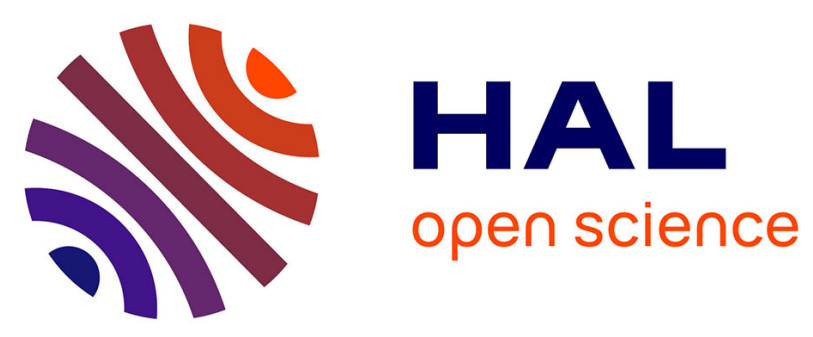

\title{
Compositional variations of chromian spinels from peridotites of the Spontang ophiolite complex, Ladakh, NW Himalayas, India: petrogenetic implications
}

\author{
Mallika K Jonnalagadda, Nitin R Karmalkar, Mathieu Benoit, Michel \\ Grégoire, Raymond Duraiswami, Shivani Harshe, Sagar Kamble
}

\section{To cite this version:}

Mallika K Jonnalagadda, Nitin R Karmalkar, Mathieu Benoit, Michel Grégoire, Raymond Duraiswami, et al.. Compositional variations of chromian spinels from peridotites of the Spontang ophiolite complex, Ladakh, NW Himalayas, India: petrogenetic implications. Geosciences Journal, 2019, 23 (6), pp.895-915. 10.1007/s12303-019-0001-3 . hal-02360235

\section{HAL Id: hal-02360235 \\ https://hal.science/hal-02360235}

Submitted on 29 Nov 2020

HAL is a multi-disciplinary open access archive for the deposit and dissemination of scientific research documents, whether they are published or not. The documents may come from teaching and research institutions in France or abroad, or from public or private research centers.
L'archive ouverte pluridisciplinaire $\mathbf{H A L}$, est destinée au dépôt et à la diffusion de documents scientifiques de niveau recherche, publiés ou non, émanant des établissements d'enseignement et de recherche français ou étrangers, des laboratoires publics ou privés. 


\title{
Compositional variations of chromian spinels from per- idotites of the Spontang ophiolite complex, Ladakh, NW Himalayas, India: petrogenetic implications
}

\author{
Mallika K. Jonnalagadda ${ }^{1 *}$, Nitin R. Karmalkar ${ }^{1}$, Mathieu Benoit ${ }^{2}$, Michel Gregoire ${ }^{2}$, Raymond A. \\ Duraiswami ${ }^{1}$, Shivani Harshe ${ }^{1}$, and Sagar Kamble ${ }^{1}$ \\ ${ }^{1}$ Department of Geology, Savitribai Phule Pune University, Pune 411007, India \\ ${ }^{2}$ Géosciences Environnement Toulouse, CNRS-IRD-Université Paul Sabatier, Observatoire Midi Pyrénées, 31400 Toulouse, France
}

\begin{abstract}
The Spontang ophiolite complex exposed along the Indus Tsangpo Suture Zone (ITSZ) represents a fragment of oce- anic lithosphere emplaced after the closure of the Neo-Tethyan Ocean. The complex lying south of the ITSZ forms the highest tec- tonic thrust slice above the Mesozoic-Early Tertiary continental margin in the Ladakh-Zanskar Himalaya. The complex consists of a well-preserved ophiolite sequence dominated by peridotites, gabbros and ultramafic cumulates along with highly tectonized sheeted dykes and pillow lavas. The mantle suite of rocks is represented by minor lherzolites, harzburgites and dunites. Chromian spinel is brown to reddish, and its morphology and textural relationship with coexisting silicates varies with strain. Spinel occurs as blebs and vermicular exsolutions within orthopyroxene to spherical inclusions within olivine, characteristic of which is the elon- gate holly leaf shape. Chrome spinels are characterized by low $\mathrm{TiO}_{2}$ and high $\mathrm{Cr}_{2} \mathrm{O}_{3}$ indicative of their depleted nature. $\mathrm{Cr} \#[=$ atomic ratio $\mathrm{Cr} /(\mathrm{Cr}+\mathrm{Al})]$ in the studied spinels are characterized by a small decrease in $\mathrm{TiO}_{2}$ for a larger increase in $\mathrm{Cr} \#$ consistent with observations for spinels aligned along the Luobusa trend of the Yarlung Zangpo Suture Zone (YZSZ) ophiolites. Variations in Cr-spinel Cr\# and Mg\# observed in the investigated peridotites may have resulted from a wide range of degrees of mantle melt- ing during their evolution. Mineral and wholerock chemistry of the Spontang peridotites is characterized by interaction between depleted magma and pre-existing oceanic lithosphere, typical of supra-subduction zone settings. The Spontang peridotites have olivine, clinopyroxene and orthopyroxene compositions similar to those from both abyssal and fore-arc peridotites and display spoon shaped REE profiles characteristic of interaction between LREE-enriched melt, derived from the subducting slab and LREE- depleted mantle residues. Equilibration temperatures calculated for the above rocks indicate that the studied samples represent typical mantle peridotites formed within the spinel stability field.
\end{abstract}

Key words: Cr-spinel, peridotite, partial melting, ophiolites, Spontang

\section{INTRODUCTION}

Remnants of a once very extensive ophiolite thrust sheet are observed to be locally preserved along the Indus Tsangpo Suture Zone (ITSZ) (Gansser, 1974; Frank et al., 1977; Srikantia and Razdan, 1980; Honegger et al, 1982; Deitrich et al, 1983; Robertson, 2000; Mahéo et al., 2004; Ahmad et al,, 2008). Ophiolites along suture zones in general are observed to occur in two tectonic settings viz. within suture itself (e.g., Xigase ophiolite, south Tibet; Nicolas et al., 1981; Göpel et al., 1984) and those obducted onto a passive continental margin (e.g., Oman ophiolite; Lippard et al., 1986). In the Ladakh-Zanskar region, two groups of ophiolites and related ophiolite mélanges are recognized viz. the North and South Ladakh group of ophiolites (Maheo et al., 2004). The North Ladakh group is represented by the Dras arc, associated with the Ladakh batholith, localized north of the ITSZ. The Spontang, Nidar and Karzog ophiolites constitute the South Ladakh group. Further east, the Indus Suture is offset dextrally 
by the Karakorum Fault but correlatives are found in Tibet as part of the Dazhuqu and Zedong terranes (Aitchison et al., 2000). Individually, these include ophiolites recorded at Jungbwa (Miller et al., 2003), Saga and Sangsang (Bédard et al., 2009), Xigaze (Nicolas et al., 1981; Girardeau et al., 1985a; Dubois-Cote et al.,

2005; Dupuis et al., 2005), Dazhuqu (Girardeau et al., 1985b; Xia et al., 2003), Zedong (McDermid et al., 2002; Aitchison et al., 2007) and Luobusa (Zhou et al., 1996). Correlatives to the west in Pakistan include Bela (Sarwar, 1992; Zaigham and Mallick,

2000), Muslim Bagh (Kakar et al., 2013) and Waziristan ophiolites

(Jan et al., 1985).

The Spontang ophiolite exposed in the Zanskar mountains of Ladakh is one of the most complete fragments of the Neo Tethys ocean that is preserved along the ITSZ (Fuchs, 1982; Reuber, 1986; Searle, 1986). The Spontang ophiolite comprises the mantle section of lherzolite and harzburgite units along with lower crustal cumulates (Riebel and Reuber, 1982; Reuber, 1986; Reuber et al.,

1992). Crustal sections are presented by gabbros, sheeted dykes and pillow lavas (Corfield and Searle, 2000). Detailed field mapping, combined with geochemical analysis carried out by Corfield et al. (2001) has helped define two major units: a full ophiolite sequence (Spontang ophiolite) overlain by an upper unit consisting of 1500-m-thick basalt-andesite volcanic and volcano-sedimentary rocks of island arc affinity (Spong arc). Previous studies carried out on the complex has focused on regional structure and timing of ophiolite obduction (Searle, 1986; Colchen et al.,

1987; Corfield and Searle, 2000) along with stratigraphy of underlying passive margin sediments (Fuchrezs, 1982; Gaetani and Garzanti, 1991). Reconnaissance mapping of the high ground of the ophiolite itself was carried out by Reibel and Reuber (1982), followed by more detailed work on the ultramafic ophiolitic rocks (Reuber, 1986) and the ophiolitic mélanges (Reuber et al., 1992; Corfield et al., 1999). U-Pb zircon ages of a diorite yielding $177 \pm 1 \mathrm{Ma}$, and an andesite of

$88 \pm 5 \mathrm{Ma}$ age have been reported by Pedersen et al. (2001),

where the latter is associated with the Spong arc sequence overlying the ophiolite. Pedersen et al. (2001) interpret the older ages to represent the Neo-Tethyan ocean crust on top of which the Spong arc developed, while the andesitic age of $\sim 88$

Ma represents the minimum age of subduction initiation to form the Spong arc.

In this paper, we present for the first-time detailed mineral

chemistry and whole rock analyses of the peridotites of the Spontang ophiolite. In addition to the above, morphological and chemical variations of accessory chromian spinels along with Cr-spinel compositions have been used to provide insights into the genesis and tectonic origin of the studied peridotites.

\section{GEOLOGICAL SETTING}

\subsection{Spontang Massif}

The Spontang klippe lying $30 \mathrm{kms}$ to the south of the ITSZ (Fuchs, 1979; Reuber, 1986; Searle, 1986) forms the highest level of the allochthon above the accretionary complex and the marine sediments of the Lamayuru complex. This area is situated between

4000 to $6000 \mathrm{mts}$ elevation near the village Photoksar and is observed to be emplaced above the Zanskar shelf sediments along the passive continental margin of the Indian plate (Corfield et al., 1999; Corfield and Searle, 2000). The complex can be subdivided into two parts viz. a western mantle unit (Reuber et al., 1986a,

1986b; Corfield et al., 2001) and an eastern crustal unit (Corfield et

al., 1999, 2001; Pedersen et al., 2001). The ophiolite sequences tectonically overlie a volcano-sedimentary mélange of late Cretaceous to early Eocene age, which contain ophiolitic blocks, and a serpentinite base (Colchen and Reuber, 1986; Colchen et al.,

1987) (Fig. 1). The complete assemblage is observed to be thrust over sedimentary series of the Zanskar unit of lower Eocene age (Colchen and Reuber, 1987; Kelemen et al., 1988). Corfield et al. (2001) were the first to recognize and describe the complete Penrose-style ophiolite suite at Spontang. Although the well- preserved ophiolite suite has been identified, the sequence has been significantly disrupted by faulting. In the present study, field studies have been carried out along 2 major traverses which expose the mantle (the Spong Valley, the Photang Valley) and crustal (the Marling chu and Bumiktse valleys) sections of the ophiolite. Detailed field studies identify an upper lherzolitic unit containing clinopyroxene and a lower, more depleted harzburgitic unit (Reuber, 1986), well exposed at the river confluence in the Spong Valley near the upper portions of the Sirsir La peak (5550 m) (Fig. 2a). Boulders of peridotite are commonly observed showing presence of chromite veins (Figs. 2b and c). Gabbroic dykes are observed to intrude into the harzburgitic unit consistent with reports by Corfield et al. (2001) (Fig. 2d). Along with several dykes, lenses of dunites are also commonly observed within the peridotite units (Fig. 2e). Structurally, three deformation events as described by Reuber (1986) are observed to affect the peridotites exposed in the Spong valley. The $S_{1}$ deformation is seen as a foliation that is ubiquitous in the peridotites, although very weak and often indiscernible in the field. Several sub-vertical NE-SW trending sinistral shear zones define the $S_{2}$ deformation. The third deformation event is recorded involving a major sub- horizontal shear zone of 20$70 \mathrm{~m}$ width, superposing the upper clinopyroxene rich lherzolitic layer from the lower more depleted harzburgitic unit.

At the base of the massive Photang Kangri peak along the 
Fig. 1. (a) Regional geological map of the Himalayas showing location of the ophiolite sequences (after Hébart et al., 2012). The study area has been demarcated by a box. (b) Detailed geological map of the Spontang ophiolite showing the major tectonic units (after Corfield et al., 2001). Stars indicate sample locations of the ultramafic samples.
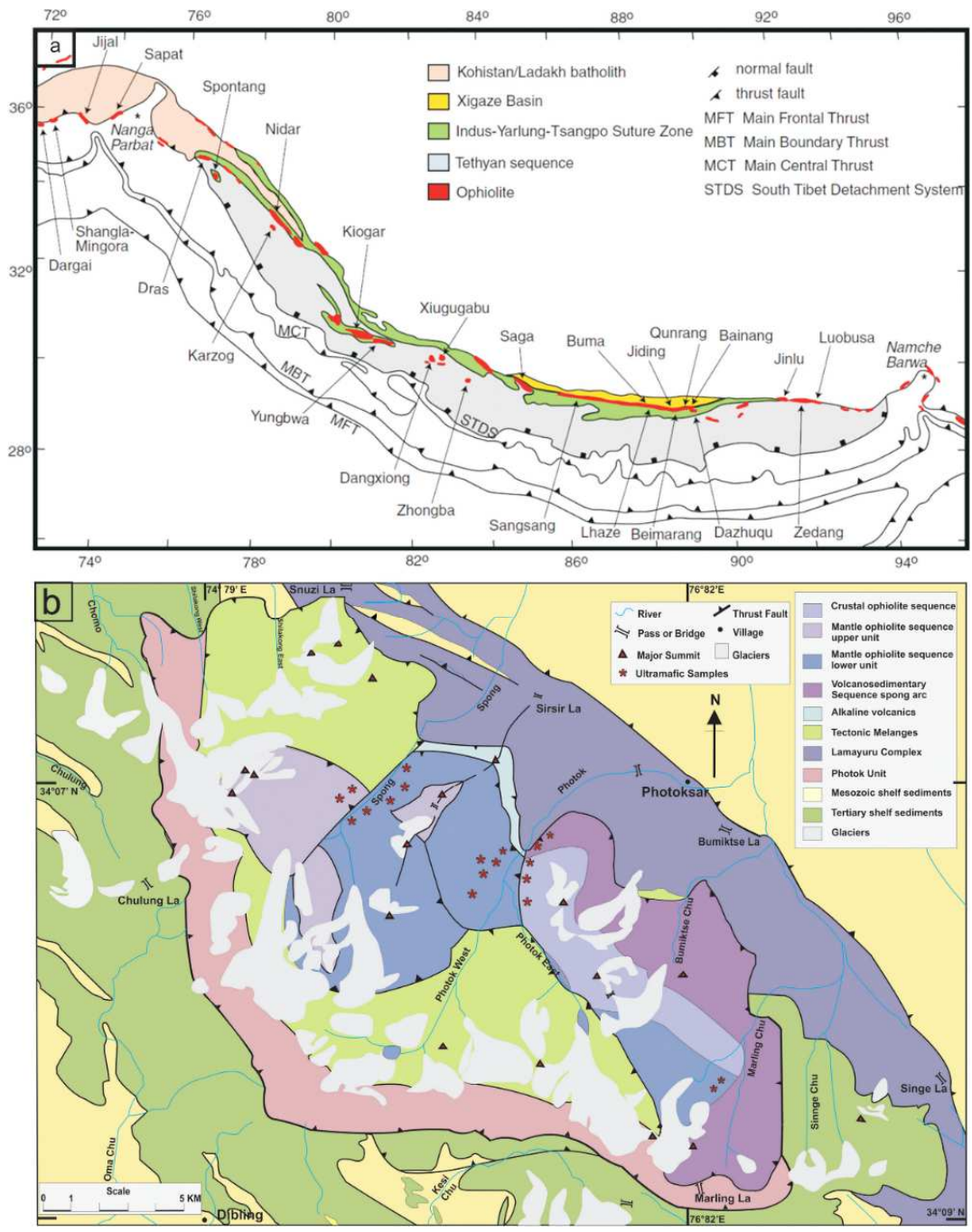

Fig. 2. Field photographs of the Spontang ophiolite: (a) Peridotites exposed along the near the river confluence in the upper Spong Valley. (b) Peridotites occurring as boulders. (c) Note the presence the chromite bands in the peridotites. (d) Gabbroic dykes intruding peridotites exposed in the Spong valley. (e) Dunite bodies are observed intercalated within the harzburgite units. (f) Pillow basalts forming boulder out- crops exposed in the Phu/Photang valley. (g) Tectonized fine grained gabbro outcrops observed in the Bumikste valley. (h) Volcano-clastic chert outcrops exposed in the Bumikste valley. 

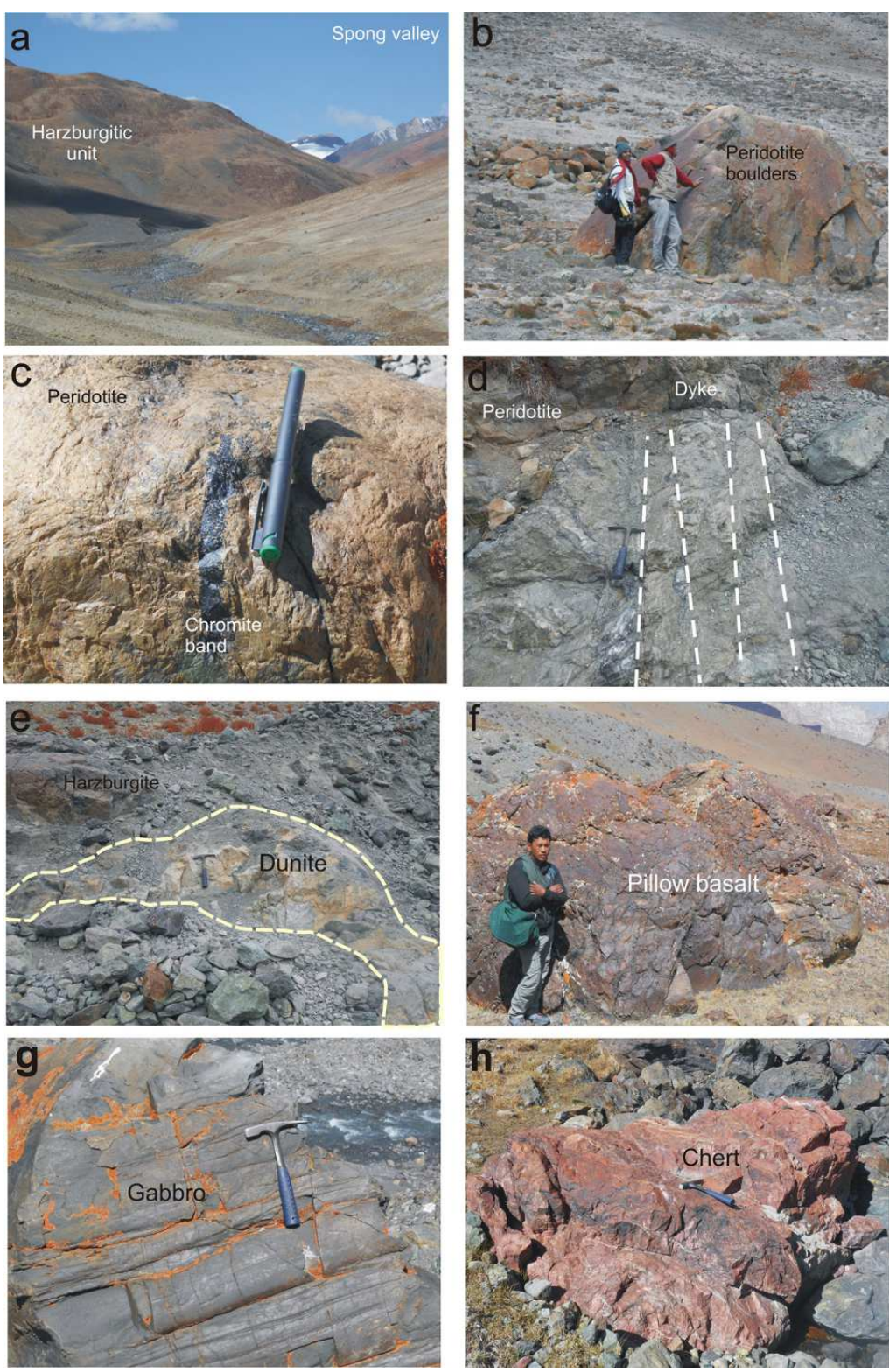
eastern flank of the Photang valley, lower crustal cumulates

2f). Medium grained basaltic dykes are observed including wehrlites, pyroxenites, gabbros and pillow lavas are exposed (Fig.

exhibiting a gradational contact with the pillow lavas.

Corfield et al. (2001)

\section{PETROGRAPHY}

the mid-Photang Valley at a higher elevation, however attempts to locate this body were unsuccessful.

The Marling chu valley and the Bumiktse valley primarily expose lower crustal sequences viz. weathered cumulates of wehrlites and pyroxenites with associated gabbros. The gabbros in the Bumiktse valley exposed below the snout of the glacier are highly tectonized and are thrust directly onto the volcano- sedimentary sequences (Fig. $2 \mathrm{~g}$ ). According to Corfield et al. (2001), a complete sequence through the Moho is preserved in the higher levels between the Bumiktse and Marling valleys forming a distinctive horizon separating the upper crustal cumulate sequence from the lower mantle sequence. Field examination at the abovementioned location however did not reveal any direct contact between the two units. Exposures in the Marling chu and Bhumiktse valleys revealed only crustal sections continuing across the inaccessible vertical cliffs and glaciers of the Photang Kangri.

\subsection{Spong Arc}

The volcano-sedimentary sequence of the Spong Island Arc comprising pillow lavas and interbedded volcanoclastic deposits are exposed at the northern edge of the Photang Kangri and in the Bumiktse and Marling chu valleys. No clear defined contact is observed between the arc deposits and the ophiolite sequence however, a transition can be observed based on the onset of volcaniclastic deposits in the field. The sequence comprises several 100 meters thick unit, consisting of angular and poorly sorted clasts, ranging widely in composition with occasional chert is exposed at Bumiktse valley (Fig. 2h). Previous studies have mapped the Spong volcanoclastic rocks as the Dras Volcanics (Srikantia and Razdan, 1981; Fuchs, 1982; Keleman and Sonnenfeld, 1983; Searle et al., 1988) however, detailed studies by Reuber et al. (1992); Corfield et al. (2001), have implied that the Spong volcanic sequence tectonically overlies the Spontang ophiolite and could be related to an immature island arc forming above the oceanic subtrate of the Spontang ophiolite.
Detailed petrographic study for the peridotite samples have been carried out and are represented by minor lherzolites, harzburgites and dunites. Textural studies of the studied peridotite rocks were carried out after Mercier and Nicolas (1975) and major textural classification is based on inclusions of accessory spinels in porphyroclasts of major minerals. Modal analysis of the studied peridotites was carried out and is depicted in Figure 3 and provided in Table 1.

Lherzolite samples show textural transition from

porphyroclastic

to equigranular mosaic texture with more than 5 vol\% clinopyroxene (after Mercier and Nicolas, 1975) and display feeble alteration in the form of presence of serpentine veins and cracks. Olivine occurs as porphyroclasts ( 0.3 to $1.5 \mathrm{~mm}$ ) set within groundmass of subhedral to anhedral grains as mosaic crystals with curved boundaries. Both generations show curvilinear, serrated boundaries, recrystallization, and presence of kink bands (Fig. 4a). Clinopyroxene may occurs as porphyroblasts or as isolated grains (Fig. 4b) within the olivine matrix with exsolution lamellae of orthopyroxenes.

Fig. 3. Modal classification of ultramafic rocks based on the propor- tions of olivine $(\mathrm{Ol})$, orthopyroxene (Opx), clinopyroxene (Cpx) (after Streckeisen, 1973).

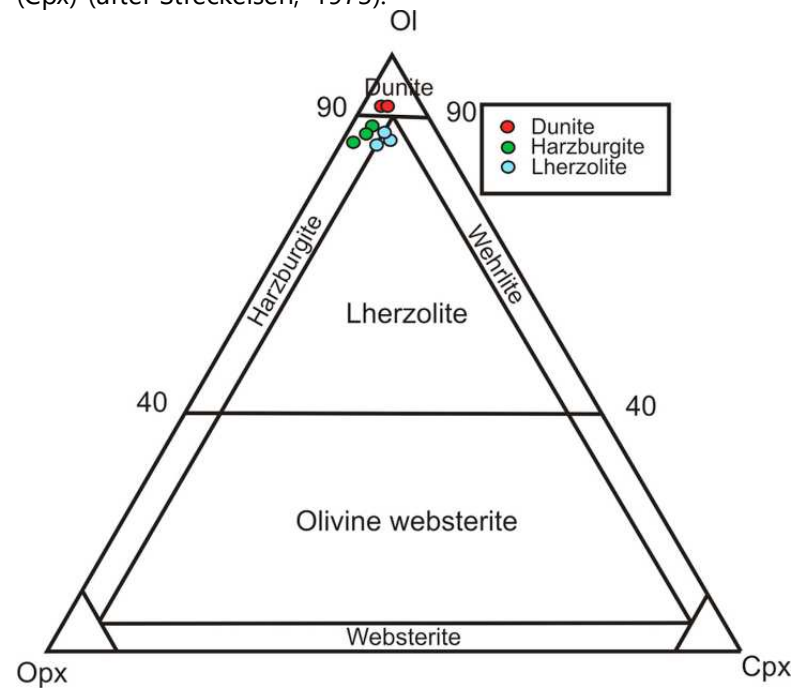


Table 1. Summary of sample details and modal abundances of the peridotites from the Spontang ophiolite complex

\begin{tabular}{|c|c|c|c|c|c|c|c|c|c|c|}
\hline Sr. No & Sample Number & Rock Type & Latitude & Longitude & Altitude (m) & Olivine & Orthopyroxene & Clinopyroxene & Spinel & Total \\
\hline 1 & B2 & Lherzolite & $34^{\circ} 06^{\prime} 756^{\prime \prime}$ & $76^{\circ} 46^{\prime} 512^{\prime}$ & 4512 & 76.8 & 12.9 & 8.5 & 1.7 & 99.9 \\
\hline 2 & B16 & Harzburgite & $34^{\circ} 07^{\prime} 412^{\prime \prime}$ & $76^{\circ} 46^{\prime} 812^{\prime}$ & 4455 & 79.7 & 13.8 & 3.8 & 2.5 & 99.8 \\
\hline 3 & E1 & Harzburgite & $34^{\circ} 06^{\prime} 591^{\prime \prime}$ & $76^{\circ} 46^{\prime} 645^{\prime}$ & 4526 & 78.9 & 13.08 & 4.26 & 2.9 & 99.1 \\
\hline 4 & E4 & Harzburgite & $34^{\circ} 06^{\prime} 190^{\prime \prime}$ & $76^{\circ} 46^{\prime} 044^{\prime}$ & 4636 & 80.13 & 16.41 & 2.60 & 0.86 & 100.0 \\
\hline 5 & E5 & Dunite & $34^{\circ} 06^{\prime} 143^{\prime \prime}$ & $76^{\circ} 45^{\prime} 994^{\prime}$ & 4640 & 82.2 & 14.5 & 0.75 & 2.5 & 99.95 \\
\hline 6 & E7 & Harzburgite & $34^{\circ} 06^{\prime} 143^{\prime \prime}$ & $76^{\circ} 45^{\prime} 994^{\prime}$ & 4642 & 83.8 & 12.3 & 1.8 & 2.1 & 100.0 \\
\hline 7 & I7 & Harzburgite & $34^{\circ} 06^{\prime} 210^{\prime \prime}$ & $34^{\circ} 06^{\prime} 210^{\prime}$ & 4607 & 80.2 & 15.5 & 3.4 & 0.8 & 99.99 \\
\hline 8 & SP3 & Dunite & $34^{\circ} 06^{\prime} 410^{\prime \prime}$ & $76^{\circ} 46^{\prime} 766^{\prime}$ & 4654 & 88.2 & 9.4 & 1.0 & 1.3 & 99.9 \\
\hline
\end{tabular}


Fig. 4. Photomicrographs of mantle peridotites of the Spontang ophiolite: (a) Olivine porphyroblasts showing undulose extinction, curvi- linear and serrated boundaries, recrystallization, and presence of kink bands (BXN). (b) Harzburgite showing porphyroclastic textures. Note the presence of pale green colored clinopyroxene (diopside) exhibiting sutured boundaries (PPL). (c) Orthopyroxene grains showing pres- ence of exsolution lamellae set within an olivine matrix (BXN). (d) Porphyroclasts of orthopyroxene showing exsolution lamellae of spinel (PPL). (e) Dark brown xenomorphic spinel showing characteristic vermicular shape with lobate boundaries in harzburgite (PPL). (f) Holly leaf spinel with opaque boundaries in harzburgite (PPL). (g) Subhedral spinel occurring as inclusions within orthopyroxene porphyroclasts (PPL). (h) euhedral spinel seen in dunite (PPL). (i) X-ray maps for the spinel grains viz. Cr, Al.
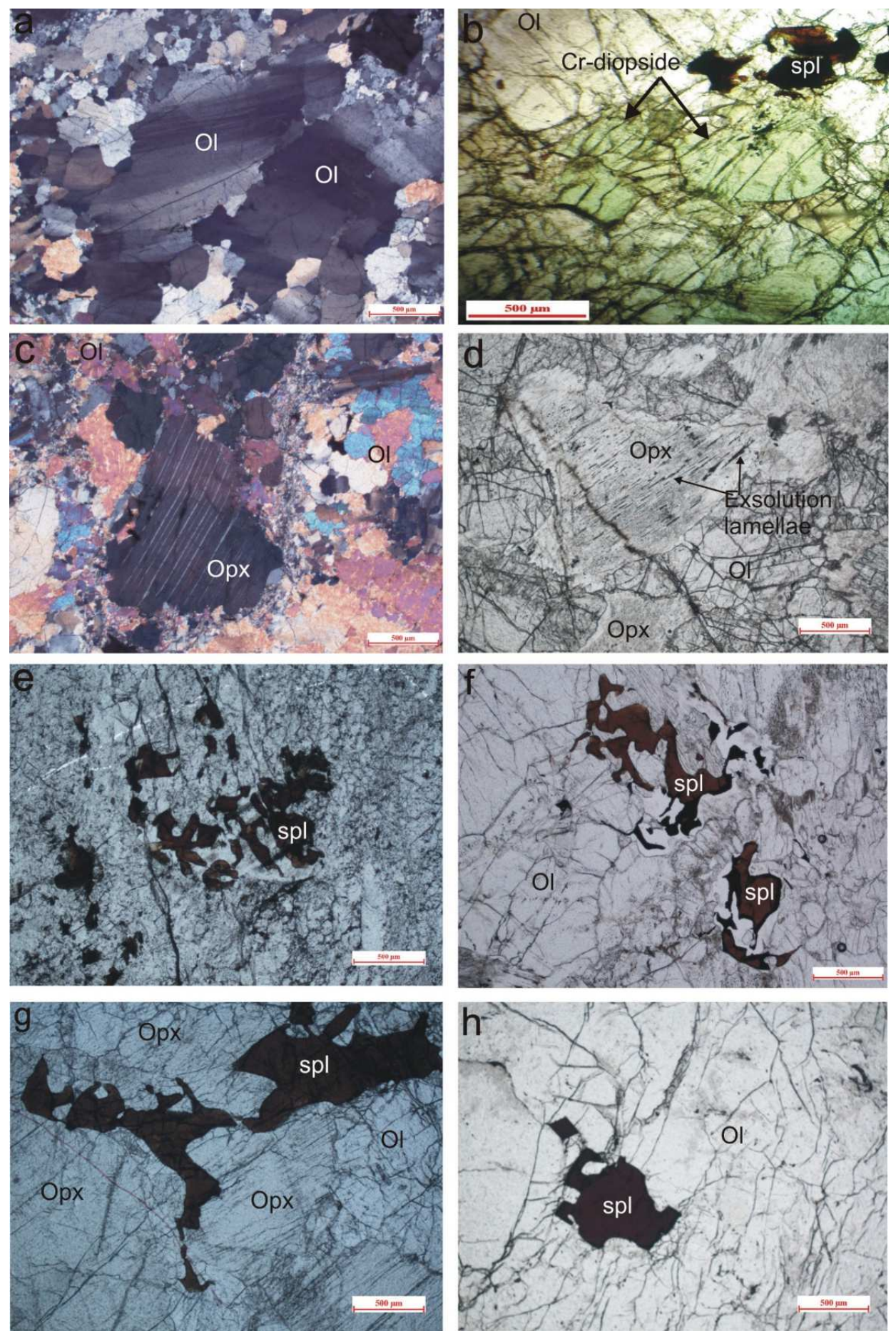
Fig. 4. (continued).

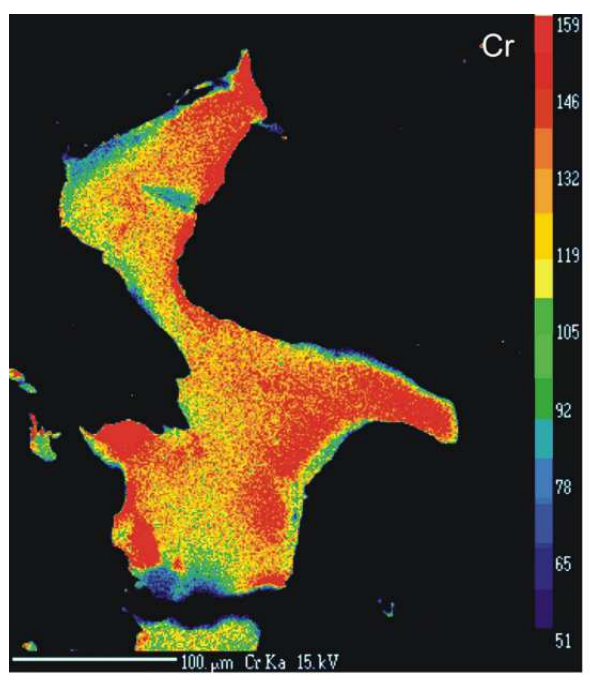

Exsolution lamellae of clinopyroxene within the orthopyroxenes are observed at places (Fig. 4c).

Harzburgite samples show protogranular to porphyroclastic textures. Their primary modal mineralogy includes olivine, orthopyroxenes, minor relicts of clinopyroxene and accessory spinel. Olivine grains are coarse, exhibit undulose extinction surrounded by small grained olivine crystals and display curvilinear and serrated boundaries meeting at triple junctions at $120^{\circ}$. Zoning, kink bands and recrystallization are commonly observed. Orthopyroxenes are colorless, usually occur as large prismatic crystals, 0.2 to $3.5 \mathrm{mms}$ in size with curvilinear boundaries and often display diopside exsolution lamellae, kink bands and gliding (Fig. 4d). Minor clinopyroxenes are small, 0.1 to $0.3 \mathrm{mms}$ in size and display pale green color in plane polarized light exhibiting sutured boundaries.

Dunites are fresh unalerted with granular texture. Olivine grains are large, porphyroclastic, elongated and display undulose extinction and intense zoning. They range in size from 0.9 to $4.00 \mathrm{~mm}$ and are surrounded by smaller subhedral grains. Matrix of olivines are subhedral to anhedral and 0.5 to $2 \mathrm{~mm}$ in size, display polygonization and serrated boundaries. Euhedral spinels are commonly observed along olivine borders and as inclusions within olivines.

Spinels are light brown picotites to dark brown colored chromium

rich and constitute up to $0.5 \mathrm{vol} \%$ to $3 \mathrm{vol} \%$ of the studied rocks. Grain sizes vary within samples, but most are between $0.2 \mathrm{~mm}$ and $1 \mathrm{~mm}$. Morphologically spinel is observed to exhibit various shapes in relationship with coexisting silicates and these shapes

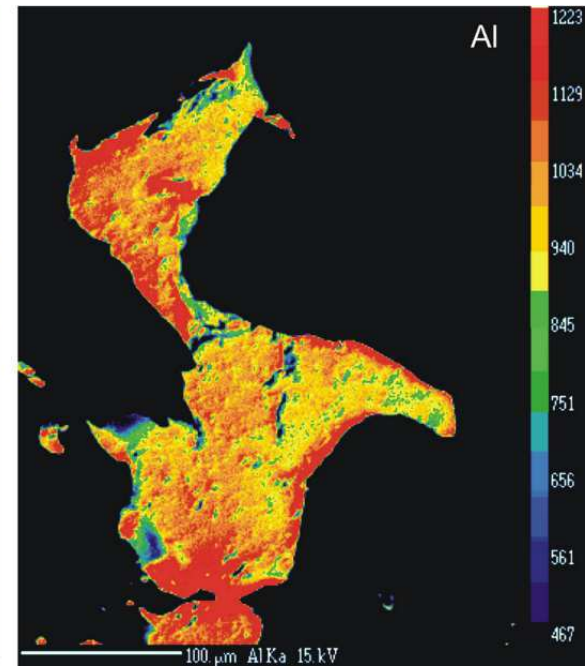

vary with the levels of strain. Spinel in the harzburgite samples occur as xenomorphic, vermicular intergrowths with lobate boundaries (Fig. 4e). The characteristic holly leaf shape is also observed in these samples (Fig. 4f). Intergrowths of orthopyroxene and spinel can be sporadically seen in these samples. At other places, subhedral spinel occurs as inclusions within orthopyroxene porphyroclasts (Fig. 4g). Spinels in dunites are mainly anhedral and occur at olivine boundaries and are rarely seen as inclusions within olivines. Euhedral idiomorphic spinels are occasionally seen to occur as inclusions within olivine grains (Fig. 4h). Under the microscope, few grains show irregular zoning due to replacement by an opaque phase along grain boundaries (Fig. 4f). This zoning is apparent in back-scattered electron images (BSE), in which the edges of the zoned Cr-spinel grains appear to be bright and separated from the inner part of the grains by sharp contacts. Mapping of a single $\mathrm{Cr}$-spinel grain shows $\mathrm{Al}-$ $\mathrm{Cr}$ zoning with distinct $\mathrm{Al}$ rich-Cr poor and $\mathrm{Al}$ poor-Cr rich regions (Fig. 4i). The observed zoning in the spinel grain is interpreted as a possible result of deformation explained by stress-directed lattice diffusion of $\mathrm{Al}$ and $\mathrm{Cr}$ (Ozawa, 1989).

\section{ANALYTICAL METHODS}

Representative mineral analyzes of spinels, pyroxenes and olivines of the investigated peridotites (harzburgites, lherzolites and dunites) of the Spontang ophiolite are given in Table 2. A Cameca SX-50 microprobe at Department of Geology, Institute of Science, Banaras Hindu University was employed to obtain 
Table 2. Representative electron microanalysis (wt\%) and atomic proportions of Cr-spinels, olivines and pyroxenes from peridotites of the

Spontang ophiolite complex

\begin{tabular}{|c|c|c|c|c|c|c|c|c|}
\hline Mineral & & & & Spi & & & & \\
\hline Sample & B2 & B16 & E1 & $\mathrm{E} 4$ & E5 & E7 & I7 & SP3 \\
\hline Rock Type & $\begin{array}{l}\text { Lherzolite } \\
\quad n=7\end{array}$ & $\begin{array}{l}\text { Harzburgite } \\
\qquad \mathrm{n}=5\end{array}$ & $\begin{array}{l}\text { Harzburgite } \\
n=15\end{array}$ & $\begin{array}{l}\text { Harzburgite } \\
\mathrm{n}=16\end{array}$ & $\begin{array}{l}\text { Dunite } \\
\mathrm{n}=3\end{array}$ & $\begin{array}{l}\text { Harzburgite } \\
\mathrm{n}=11\end{array}$ & $\begin{array}{l}\text { Harzburgite } \\
\quad \mathrm{n}=3\end{array}$ & $\begin{array}{l}\text { Dunite } \\
\mathrm{n}=10\end{array}$ \\
\hline $\mathrm{SiO}_{2}$ & 2.47 & 0.01 & - & - & - & 0.03 & 0.12 & 0.17 \\
\hline $\mathrm{TiO}_{2}$ & 0.09 & 0.07 & 0.07 & 0.11 & 0.05 & 0.02 & 0.13 & 0.11 \\
\hline $\mathrm{Al}_{2} \mathrm{O}_{3}$ & 27.12 & 39.62 & 42.34 & 40.40 & 22.63 & 45.76 & 29.90 & 29.76 \\
\hline $\mathrm{Cr}_{2} \mathrm{O}_{3}$ & 40.07 & 27.68 & 23.92 & 26.20 & 42.76 & 21.90 & 38.82 & 34.78 \\
\hline $\mathrm{FeO}$ & 15.98 & 15.77 & 14.63 & 13.78 & 19.56 & 13.80 & 15.58 & 18.49 \\
\hline $\mathrm{MgO}$ & 11.96 & 15.19 & 16.30 & 16.56 & 11.95 & 16.92 & 14.56 & 13.35 \\
\hline $\mathrm{MnO}$ & 0.32 & 0.14 & 0.27 & 0.26 & 0.42 & 0.12 & 0.23 & 0.24 \\
\hline $\mathrm{NiO}$ & 0.14 & - & 0.16 & 0.15 & 0.29 & - & - & 0.34 \\
\hline $\mathrm{CaO}$ & 0.16 & 0.03 & - & - & 0.06 & 0.03 & 0.01 & 0.13 \\
\hline $\mathrm{SrO}$ & 1.63 & - & 0.89 & 1.04 & 1.72 & - & - & 1.44 \\
\hline Total & 99.88 & 98.69 & 99.49 & 99.52 & 99.44 & 98.72 & 99.49 & 99.01 \\
\hline $\mathrm{Ti}$ & 0.00 & 0.00 & 0.00 & 0.00 & 0.00 & 0.00 & 0.00 & 0.00 \\
\hline $\mathrm{Al}$ & 1.01 & 1.34 & 1.42 & 1.36 & 0.84 & 1.49 & 1.05 & 1.03 \\
\hline $\mathrm{Cr}$ & 1.00 & 0.63 & 0.54 & 0.59 & 1.06 & 0.49 & 0.91 & 0.87 \\
\hline $\mathrm{Fe}^{3+}$ & 0.07 & 0.03 & 0.05 & 0.05 & 0.09 & 0.03 & 0.04 & 0.09 \\
\hline $\mathrm{Fe}^{2+}$ & 0.43 & 0.35 & 0.30 & 0.29 & 0.42 & 0.31 & 0.35 & 0.40 \\
\hline $\mathrm{Mn}$ & 0.01 & 0.00 & 0.01 & 0.01 & 0.01 & 0.69 & 0.01 & 0.01 \\
\hline $\mathrm{Mg}$ & 0.56 & 0.65 & 0.69 & 0.71 & 0.56 & 0.00 & 0.64 & 0.58 \\
\hline Total & 3.00 & 3.00 & 3.00 & 3.00 & 3.00 & 3.00 & 3.00 & 3.00 \\
\hline $\mathrm{Cr} \#$ & 0.50 & 0.62 & 0.27 & 0.30 & 0.53 & 0.24 & 0.46 & 0.44 \\
\hline Mg\# & 0.57 & 0.34 & 0.69 & 0.71 & 0.57 & 0.69 & 0.65 & 0.59 \\
\hline Mineral & & & & Oliv & & & & \\
\hline Sample & B2 & B16 & E1 & E & & E7 & I7 & SP3 \\
\hline Rock Type & $\begin{array}{c}\text { Lherzolite } \\
\mathrm{n}=7\end{array}$ & $\begin{array}{l}\text { Harzburgite } \\
n=3\end{array}$ & $\begin{array}{l}\text { Harzburgite } \\
\mathrm{n}=11\end{array}$ & $\begin{array}{l}\text { Harzb } \\
\mathrm{n}=\end{array}$ & & $\begin{array}{c}\text { Harzburgite } \\
\mathrm{n}=11\end{array}$ & $\begin{array}{l}\text { Harzburgite } \\
n=3\end{array}$ & $\begin{array}{l}\text { Dunite } \\
\mathrm{n}=21\end{array}$ \\
\hline $\mathrm{SiO}_{2}$ & 42.61 & 41.45 & 45.74 & 41 & & 47.00 & 43.35 & 44.11 \\
\hline $\mathrm{FeO}$ & 8.07 & 8.78 & 7.94 & 9. & & 8.03 & 8.21 & 8.88 \\
\hline $\mathrm{MgO}$ & 47.61 & 48.96 & 44.05 & 48 & & 42.84 & 47.28 & 46.17 \\
\hline $\mathrm{MnO}$ & 0.19 & 0.09 & 0.17 & 0 . & & 0.10 & 0.10 & 0.10 \\
\hline $\mathrm{NiO}$ & 0.39 & - & 0.21 & 0.2 & & - & - & 0.31 \\
\hline $\mathrm{Cr}_{2} \mathrm{O}_{3}$ & 0.19 & 0.15 & 0.24 & 0. & & 0.34 & 0.14 & 0.17 \\
\hline $\mathrm{CaO}$ & - & 0.03 & - & - & & 0.21 & 0.10 & - \\
\hline Total & 99.64 & 99.53 & 99.47 & 100 & & 99.45 & 99.50 & 100.02 \\
\hline $\mathrm{Si}$ & 1.06 & 1.02 & 1.15 & 1. & & 1.19 & 1.07 & 1.10 \\
\hline $\mathrm{Fe}^{3+}$ & 0.02 & - & 0.02 & $0 . \mathrm{C}$ & & - & - & 0.02 \\
\hline $\mathrm{Fe}^{2+}$ & 0.15 & 0.18 & 0.15 & 0.1 & & 0.17 & 0.17 & 0.16 \\
\hline $\mathrm{Mn}$ & 0.00 & 0.00 & 0.00 & 0.0 & & 0.00 & 0.00 & 0.00 \\
\hline $\mathrm{Mg}$ & 1.75 & 1.79 & 1.64 & 1.7 & & 1.60 & 1.74 & 1.70 \\
\hline $\mathrm{Ca}$ & 0.01 & 0.00 & - & - & & 0.01 & 0.00 & 0.00 \\
\hline$\% \mathrm{Fo}$ & 90.61 & 90.73 & 90.62 & 90. & & 89.96 & 90.82 & 90.09 \\
\hline$\% \mathrm{Fa}$ & 8.65 & 9.13 & 9.18 & 9.4 & & 9.52 & 8.89 & 9.73 \\
\hline $\mathrm{Ca}-\mathrm{Ol}$ & 0.53 & 0.04 & - & - & & 0.40 & 0.17 & 0.07 \\
\hline
\end{tabular}

mineral chemistry data of the studied rocks. The for electron probe micro analyses using LEICA-EM ACE200 accelerating voltage and beam current were $15 \mathrm{kV}$ and $10 \mathrm{nA}$ instrument. Natural (fluorite, albite, halite, periclase, respectively. Polished thin sections were coated with $20 \mathrm{~nm}$ peridote, corundum, wollastonite, apatite pyrite, orthoclase, thin layer of carbon rutile, chromite, 
Table 2. (continued)

\begin{tabular}{|c|c|c|c|c|c|c|c|c|c|}
\hline \multirow{2}{*}{$\begin{array}{l}\text { Mineral } \\
\text { Sample }\end{array}$} & \multicolumn{5}{|c|}{ Clinopyroxene } & \multicolumn{4}{|c|}{ Orthopyroxene } \\
\hline & B2 & B16 & E7 & I7 & SP3 & B16 & E7 & I7 & SP3 \\
\hline Rock Type & $\begin{array}{c}\text { Lherzolite } \\
n=14\end{array}$ & $\begin{array}{l}\text { Harzburgite } \\
n=5\end{array}$ & $\begin{array}{l}\text { Harzburgite } \\
n=9\end{array}$ & $\begin{array}{c}\text { Harzburgite } \\
n=10\end{array}$ & $\begin{array}{l}\text { Dunite } \\
\mathrm{n}=10\end{array}$ & $\begin{array}{l}\text { Harzburgite } \\
\quad \mathrm{n}=1\end{array}$ & $\begin{array}{l}\text { Harzburgite } \\
n=2\end{array}$ & $\begin{array}{l}\text { Harzburgite } \\
\mathrm{n}=11\end{array}$ & $\begin{array}{l}\text { Dunite } \\
\mathrm{n}=7\end{array}$ \\
\hline $\mathrm{SiO}_{2}$ & 53.14 & 52.81 & 52.88 & 53.04 & 52.74 & 56.48 & 56.21 & 55.90 & 56.12 \\
\hline $\mathrm{TiO}_{2}$ & 0.11 & 0.11 & 0.12 & 0.13 & 0.01 & 0.04 & 0.03 & 0.03 & 1.24 \\
\hline $\mathrm{Al}_{2} \mathrm{O}_{3}$ & 1.52 & 3.03 & 2.90 & 3.12 & 5.27 & 2.35 & 2.67 & 3.11 & 0.56 \\
\hline $\mathrm{Cr}_{2} \mathrm{O}_{3}$ & 0.65 & 1.03 & 0.78 & 1.27 & 0.96 & 0.56 & 0.71 & 0.91 & 0.46 \\
\hline $\mathrm{FeO}$ & 1.67 & 2.25 & 2.04 & 2.03 & 3.68 & 6.07 & 6.22 & 6.09 & 6.32 \\
\hline $\mathrm{MnO}$ & 0.05 & 0.05 & 0.06 & 0.08 & 0.06 & 0.12 & 0.15 & 0.15 & 2.22 \\
\hline $\mathrm{MgO}$ & 17.44 & 16.62 & 16.55 & 16.40 & 23.90 & 33.14 & 32.92 & 32.61 & 19.86 \\
\hline $\mathrm{CaO}$ & 24.09 & 23.09 & 23.91 & 23.25 & 12.37 & 0.57 & 0.68 & 0.83 & 13.12 \\
\hline $\mathrm{K}_{2} \mathrm{O}$ & 0.04 & 0.00 & 0.00 & 0.01 & 0.00 & - & 0.01 & 0.01 & 0.01 \\
\hline $\mathrm{Na}_{2} \mathrm{O}$ & 0.18 & 0.27 & 0.13 & 0.42 & 0.33 & 0.01 & 0.01 & 0.01 & 0.07 \\
\hline $\mathrm{NiO}$ & 0.07 & - & - & - & 0.06 & - & - & - & 0.12 \\
\hline Total & 99.38 & 99.33 & 99.44 & 99.81 & 99.47 & 99.34 & 99.64 & 99.68 & 100.24 \\
\hline $\mathrm{Si}$ & 1.95 & 1.93 & 1.93 & 1.93 & 1.87 & 1.97 & 1.95 & 1.94 & 1.94 \\
\hline $\mathrm{Ti}$ & - & - & - & - & - & - & - & - & $-\mathrm{Al}$ \\
\hline & 0.07 & 0.13 & 0.13 & 0.13 & 0.24 & 0.10 & 0.11 & 0.13 & 0.07 \\
\hline $\mathrm{Cr}$ & 0.02 & 0.03 & 0.02 & 0.04 & 0.03 & 0.02 & 0.02 & 0.02 & 0.01 \\
\hline $\mathrm{Fe}^{3+}$ & 0.03 & 0.00 & - & - & 0.09 & - & - & - & 0.03 \\
\hline $\mathrm{Fe}^{2+}$ & 0.03 & 0.07 & 0.06 & 0.06 & 0.12 & 0.18 & 0.18 & 0.18 & 0.16 \\
\hline $\mathrm{Mn}$ & - & 0.00 & - & - & - & - & - & - & - \\
\hline $\mathrm{Mg}$ & 0.95 & 0.91 & 0.90 & 0.89 & 1.26 & 1.72 & 1.71 & 1.69 & 1.70 \\
\hline $\mathrm{Ca}$ & 0.95 & 0.91 & 0.94 & 0.91 & 0.47 & 0.02 & 0.03 & 0.03 & 0.11 \\
\hline $\mathrm{Na}$ & 0.01 & 0.02 & 0.01 & 0.03 & 0.03 & - & - & - & 0.01 \\
\hline Total & 4.00 & 4.00 & 4.00 & 4.00 & 4.00 & 4.00 & 4.00 & 4.00 & 4.00 \\
\hline Wo & 0.49 & 0.48 & 0.49 & 0.48 & 0.70 & 0.01 & 0.02 & 0.02 & 0.02 \\
\hline En & 0.50 & 0.48 & 0.47 & 0.47 & 0.07 & 0.90 & 0.89 & 0.89 & 0.90 \\
\hline Fs & 0.01 & 0.04 & 0.03 & 0.33 & 0.26 & 0.09 & 0.09 & 0.09 & 0.08 \\
\hline Mg\# & 0.98 & 0.92 & 0.94 & 0.94 & 0.94 & 0.91 & 0.91 & 0.91 & 0.92 \\
\hline
\end{tabular}

rhodonite, hematite, celestine, zircon and barite) and an aliquot of the final solution was taken and diluted ( $\mathrm{F}=10000)$ synthetic standards ( $\mathrm{Ni} \& \mathrm{Nb}$ and synthetic glass standard for ICP-MS measurements on the ELEMENT XR, ThermoYAG supplied by CAMECA-AMETEK) were used for calibration Scientific and proceed following the direct measurement of the machine. Data processing was carried out using SxSAB protocol from Rospabé et al. (2017). International geostandard version 6.1 and SX-Results softwares of CAMECA. Major oxides UBN and blanks were regularly run during the set (see Table were determined by X-ray fluorescence spectroscopy (XRF) 3). The blanks were subtracted to the sample and standard on pressed pellets at the Department of Geology, Savitribai signals before processing the data according to Rospabé et al. Phule Pune University. The analytical uncertainty is (2017).

estimated to be $\pm 1 \%$ for all major oxides. Trace and Rare Earth Elements (REE's) were analyzed following detailed sample preparation and analytical procedures described by Barrat et al. $(1996,2012)$ and Rospabé et al. (2017) at GETOMP, Toulouse, France. About $0.200 \mathrm{~g}$ of sample powder was accurately weighed and transferred to $22 \mathrm{ml}$ Savillex PFA beakers. An amount (between 1.5-15 ng) of Tm (in solution) was added to each sample, except to the blank and to the reference material BHVO-2. Sample digestion procedure was adopted following method Cof Yokoyama etal.(1999). After total dissolution,

\section{RESULTS}

\subsection{Whole Rock Data}

Whole-rock major elements were carried out on 6 peridotite samples and are given in Table 3. Loss on ignition (LOI) values are $<1 \%$ indicating the freshness of the samples. The Mg\# varies from 92 to 93 similar to that of modern oceanic depleted and residual peridotites (Bonatti and Michal, 1989; Bodinier and 
Table 3. Representative whole-rock, trace and REE compositions of ultramafic rocks of the Spontang ophiolite complex

\begin{tabular}{|c|c|c|c|c|c|c|c|c|}
\hline Samples & B2 & B16 & E1 & E4 & E5 & E7 & L13 & L14 \\
\hline Rock Type & Lherzolite & Harzburgite & Harzburgite & Harzburgite & Dunite & Harzburgite & UBN & UBN \\
\hline $\mathrm{SiO}_{2}$ & 45.06 & 46.04 & 44.78 & 45.09 & 44.4 & 44.57 & & \\
\hline $\mathrm{TiO}_{2}$ & nd & 0.02 & nd & 0.02 & nd & nd & & \\
\hline $\mathrm{Al}_{2} \mathrm{O}_{3}$ & 1.26 & 1.28 & 1.41 & 1.49 & 0.66 & 1.18 & & \\
\hline $\mathrm{MnO}$ & 0.14 & 0.14 & 0.14 & 0.14 & 0.14 & 0.15 & & \\
\hline $\mathrm{Fe}_{2} \mathrm{O}_{3}$ & 9.9 & 9.48 & 9.88 & 9.81 & 9.76 & 10.08 & & \\
\hline $\mathrm{CaO}$ & 1.69 & 1.19 & 1.2 & 2.08 & 0.77 & 1.15 & & \\
\hline $\mathrm{MgO}$ & 40.8 & 40.88 & 41.51 & 39.66 & 43.21 & 41.52 & & \\
\hline $\mathrm{Cr}_{2} \mathrm{O}_{3}$ & 0.47 & 0.43 & 0.38 & 0.46 & 0.44 & 0.33 & & \\
\hline $\mathrm{NiO}$ & 0.34 & 0.32 & 0.34 & 0.33 & 0.36 & 0.34 & & \\
\hline LOI & 0.35 & 0.23 & 0.35 & 0.93 & 0.26 & 0.67 & & \\
\hline TOTAL & 100.1 & 100 & 99.99 & 100.01 & 99.99 & 99.98 & & \\
\hline $\mathrm{Rb}$ & 0.020 & 0.024 & 0.021 & 0.011 & 0.062 & 0.017 & 3.34 & 3.48 \\
\hline $\mathrm{Sr}$ & 0.122 & 0.173 & 0.203 & 0.361 & 2.541 & 0.215 & 4.0 & 4.1 \\
\hline Y & 0.180 & 0.368 & 0.272 & 0.696 & 0.184 & 0.395 & 2.65 & 2.76 \\
\hline $\mathrm{Zr}$ & 0.023 & 0.037 & 0.040 & 0.049 & 0.494 & 0.068 & 3.3 & 3.5 \\
\hline $\mathrm{Nb}$ & 0.008 & 0.013 & 0.013 & 0.023 & 0.125 & 0.019 & 0.04 & 0.04 \\
\hline Mo & 0.025 & 0.022 & 0.023 & 0.012 & 0.307 & 0.022 & 0.33 & 0.33 \\
\hline $\mathrm{Ba}$ & 0.178 & 0.282 & 0.257 & 0.217 & 0.788 & 0.203 & 27.2 & 28.4 \\
\hline $\mathrm{La}$ & 0.003 & 0.015 & 0.0048 & 0.006 & 0.363 & 0.007 & 0.33 & 0.35 \\
\hline $\mathrm{Ce}$ & 0.008 & 0.006 & 0.0116 & 0.014 & 0.692 & 0.016 & 0.85 & 0.89 \\
\hline $\operatorname{Pr}$ & 0.001 & 0.001 & 0.0011 & 0.002 & 0.065 & 0.002 & 0.12 & 0.13 \\
\hline $\mathrm{Nd}$ & 0.003 & 0.004 & 0.0054 & 0.010 & 0.207 & 0.009 & 0.60 & 0.64 \\
\hline $\mathrm{Sm}$ & 0.002 & 0.005 & 0.0035 & 0.009 & 0.030 & 0.005 & 0.22 & 0.23 \\
\hline $\mathrm{Eu}$ & 0.001 & 0.002 & 0.0014 & 0.005 & 0.006 & 0.003 & 0.088 & 0.092 \\
\hline Gd & 0.006 & 0.017 & 0.0109 & 0.036 & 0.030 & 0.017 & 0.280 & 0.298 \\
\hline $\mathrm{Tb}$ & 0.002 & 0.004 & 0.0034 & 0.009 & 0.004 & 0.006 & 0.060 & 0.063 \\
\hline Dy & 0.019 & 0.040 & 0.0308 & 0.093 & 0.028 & 0.050 & 0.443 & 0.465 \\
\hline Ho & 0.006 & 0.014 & 0.0094 & 0.025 & 0.007 & 0.014 & 0.098 & 0.105 \\
\hline Er & 0.024 & 0.052 & 0.0415 & 0.089 & 0.024 & 0.055 & 0.297 & 0.312 \\
\hline $\mathrm{Yb}$ & 0.039 & 0.074 & 0.0548 & 0.112 & 0.034 & 0.074 & 0.305 & 0.324 \\
\hline $\mathrm{Lu}$ & 0.007 & 0.013 & 0.0100 & 0.020 & 0.006 & 0.012 & 0.046 & 0.050 \\
\hline $\mathrm{Hf}$ & 0.002 & 0.004 & 0.0023 & 0.008 & 0.018 & 0.006 & 0.156 & 0.171 \\
\hline $\mathrm{Ta}$ & nd & 0.000 & nd & 0.008 & 0.033 & 0.001 & 0.015 & 0.016 \\
\hline $\mathrm{Pb}$ & 0.026 & 0.010 & 0.016 & 0.012 & 0.286 & 0.017 & 16.6 & 16.9 \\
\hline Th & 0.001 & 0.001 & 0.0010 & 0.001 & 0.125 & 0.002 & 0.070 & 0.072 \\
\hline $\mathrm{U}$ & 0.003 & 0.002 & 0.0040 & 0.003 & 0.011 & 0.003 & 0.056 & 0.061 \\
\hline Sc & 9.61 & 12.03 & 11.98 & 3.87 & 7.41 & 16.66 & 12.5 & 13.0 \\
\hline $\mathrm{Ti}$ & 41.59 & 128.02 & 59.89 & 128.92 & 56.52 & 88.82 & 582 & 603 \\
\hline V & 33.98 & 49.17 & 44.21 & 62.07 & 23.19 & 38.25 & 62 & 65 \\
\hline $\mathrm{Cr}$ & $1,750.69$ & $2,184.52$ & $2,228.96$ & $2,292.79$ & $1,529.09$ & $1,226.96$ & 2516 & 2733 \\
\hline $\mathrm{Mn}$ & 851.75 & $1,004.95$ & 968.75 & $1,007.97$ & 931.31 & 891.33 & 936 & 983 \\
\hline Co & 103.44 & 125.64 & 121.31 & 128.45 & 125.87 & 109.40 & 98 & 102 \\
\hline $\mathrm{Ni}$ & $2,076.25$ & $2,352.54$ & $2,254.92$ & $2,304.09$ & $2,470.31$ & $2,121.24$ & 2026 & 2109 \\
\hline $\mathrm{Cu}$ & 9.26 & 8.98 & 12.16 & 22.48 & 4.96 & 8.06 & 23.7 & 23.7 \\
\hline $\mathrm{Zn}$ & 36.34 & 51.40 & 44.54 & 49.10 & 40.77 & 40.91 & 82 & 86 \\
\hline $\mathrm{Ga}$ & 0.59 & 1.04 & 0.88 & 1.36 & 0.46 & 0.92 & 2.32 & 2.38 \\
\hline
\end{tabular}

Godard, 2007). All peridotites are strongly depleted in and enriched in transition elements viz. Ni in the range of major elements with $\mathrm{SiO}_{2}<45$ wt $\%, \mathrm{TiO}_{2}<0.02$ wt $\%, \mathrm{CaO}<$ $2.08 \mathrm{wt} \%$

2076 to $2470 \mathrm{ppm}$ and $\mathrm{Cr}$ in the range of 1126 to 2292 ppm. MgO 

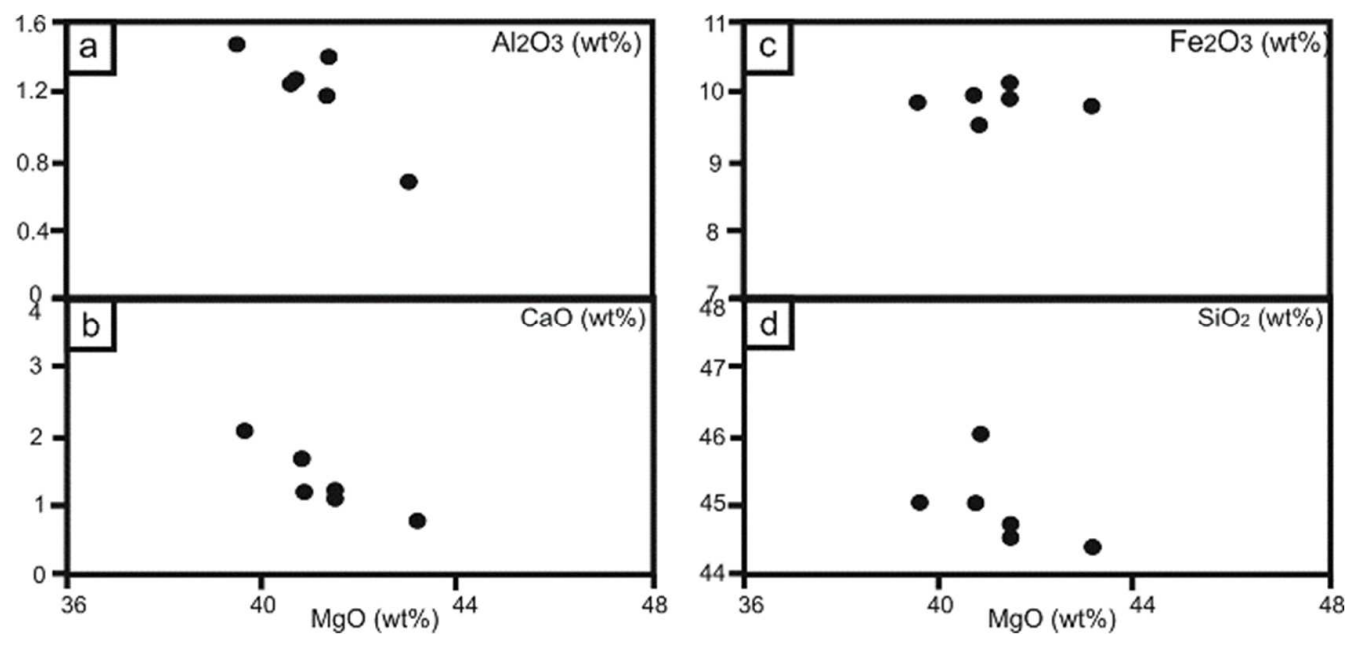
Fig. 5. Primary bulk composition of peridotites of the Spontang ophiolite complex as a function of $\mathrm{MgO}$ (a) $\mathrm{Al}_{2} \mathrm{O}_{3}$, (b) $\mathrm{CaO}_{1}$ (c) $\mathrm{Fe}_{2} \mathrm{O}_{3}$, and (d) $\mathrm{SiO}_{2}$.

contents increase systematically from lherzolite to harzburgite and dunite considered as an index of melt depletion (Parkinson and Pearce, 1998). $\mathrm{Al}_{2} \mathrm{O}_{3}$ and $\mathrm{CaO}$ contents range from $0.66 \mathrm{wt} \%$ to $1.49 \mathrm{wt} \%$ and $0.77 \mathrm{wt} \%$ to $2.08 \mathrm{wt} \%$ respectively and show an inverse correlation when plotted against $\mathrm{MgO}$ (Fig. 5). In addition to this, bulk $\mathrm{SiO}_{2}$ shows a negative correlation against $\mathrm{MgO}$ and is consistent with trends displayed by abyssal peridotites (Baker and Beckett, 1999). FeO-MgO trends are essentially horizontal and are probably attributed to abyssal peridotites having experienced extensive olivine addition by upwelling mantle melts (Kelemen et al., 1997; Niu et al., 1997; Baker and Beckett, 1999). Chondrite normalized rare earth element patterns are illustrated in Figure

6 and are characterized by extremely low REE concentrations ranging from 0.01 to 0.12 times the chondritic value. The studied samples show smooth LREE depleted patterns to positive MREE to HREE slopes indicating high degree of partial melting. The Spontang peridotites have spoon-shaped REE profiles; characteristic of interaction between tholeiitic or calc-alkaline melts and REE- depleted mantle residues. It is important to note that the dunite displays the most LREE enriched profile, that is characteristic of reactive melting (Dick, 1977a; Quick, 1981; Edwards, 1990; Kelemen,

1990; Kelemen et al., 1992; Zhou et al., 2005) and typically found in dunites sitting at mantle transition zone levels (Godard et al.,

2000; Koga et al., 2001; Rospabé et al., 2018). Lherzolite sample shows the most depleted MREE and HREE patterns as compared to the harzburgite samples. The absence of Eu anomaly indicates

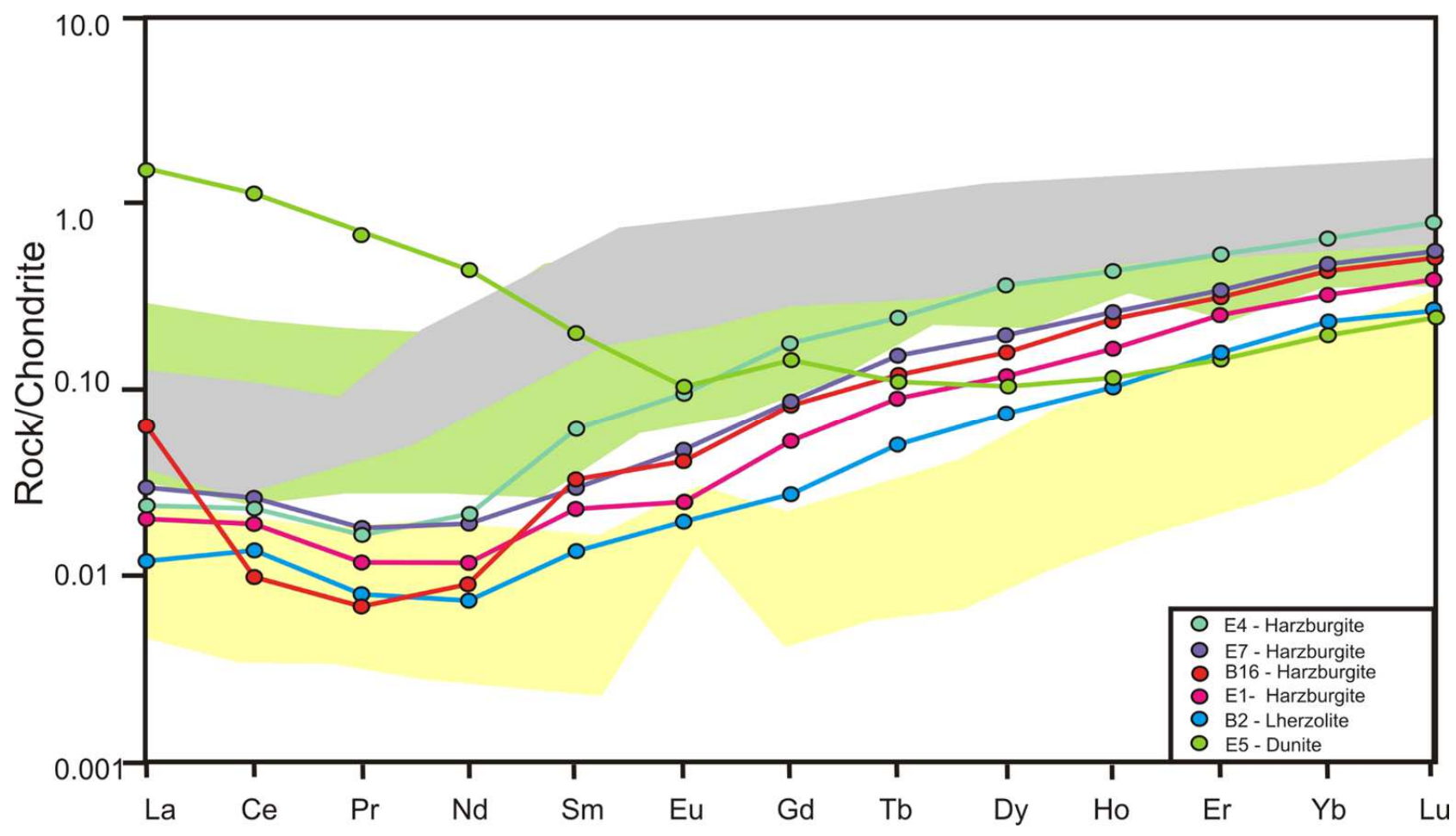

Fig. 6. Chondrite normalized REE patterns for the peridotites of the Spontang ophiolite complex. Normalizing values are after Sun and McDonough, (1989). Grey band represents composition of YSZS peridotites and are taken after Dupuis et al. (2005). Yellow and green bands represent compositions of fore-arc (after Parkinson and Pearce, 1998) and abyssal peridotites, respectively (after Singh, 2013). 
absence of plagioclase accumulation in the source.

\subsection{Spinel}

Analyzed spinels in the studied peridotites are fresh, unaltered with low $\mathrm{SiO}_{2}$ and $\mathrm{TiO}_{2}$ contents and high $\mathrm{Cr}_{2} \mathrm{O}_{3}$. Dunite samples contain $\mathrm{Cr}$-spinels with high $\mathrm{Cr} \#$ values ranging between 0.31 to as high as $0.73 . \mathrm{Cr}_{2} \mathrm{O}_{3}$ and $\mathrm{Al}_{2} \mathrm{O}_{3}$ values vary between $28 \mathrm{wt} \%$ to $51 \mathrm{wt} \%$ and $9 \mathrm{wt} \%$ to $39 \mathrm{wt} \%$. $\mathrm{FeO}$ contents vary between $18 \mathrm{wt} \%$ to $20 \mathrm{wt} \%$ with low $\mathrm{TiO}_{2}$ contents $(0.05 \mathrm{wt} \%$ to $0.11 \mathrm{wt} \%)$. The harzburgites host $\mathrm{Cr}-$ spinels with lower Cr\# (0.21 to 0.54) and Mg\# (0.42 to 0.70) relative to dunites and are classified as spinels with one sample falling on the spinel-hercynite solid solution boundary (Fig. 7a). $\mathrm{Cr}_{2} \mathrm{O}_{3}$ ranges between 19 wt\% and 43 wt $\%, \mathrm{Al}_{2} \mathrm{O}_{3}$ between $24 \mathrm{wt} \%$ and $48 \mathrm{wt} \%$. $\mathrm{FeO}$ contents are lower than dunites and range between $14 \mathrm{wt} \%$ and $16 \mathrm{wt} \%$. $\mathrm{TiO}_{2}$ ranges between $0.02 \mathrm{wt} \%$ and $0.13 \mathrm{wt} \%$. Lherzolites display Cr-spinels with the highest $\mathrm{Mg \#} \mathrm{(0.70} \mathrm{to} 0.79$ ) and lowest $\mathrm{Cr} \#(0.10$ to 0.34 ), indicating a moderately fertile character (Fig. 7a). Overall it can be said that the $\mathrm{Cr} \#$ values of $\mathrm{Cr}$-spinel increase and Mg\# values decrease simultaneously from lherzolite to harzburgite and dunite and plot along a trend, defined by a large increase in Cr\# for a relatively small decrease in Mg\#. All chromian spinels have Cr\# and Mg\# similar to those of mantle peridotites and plot in the mantle array field on the $\mathrm{Cr}_{2} \mathrm{O}_{3}$ vs. $\mathrm{Al}_{2} \mathrm{O}_{3}$ diagram (Fig. 7b). Samples when plotted in the $\mathrm{Cr}-\mathrm{Al}_{-} \mathrm{Fe}^{3+}$ triangular diagram (Fig. 7c) of Jan and Windley (1990) fall in the residual peridotite or ophiolite fields consistent with the low $\mathrm{TiO}_{2}$ contents $(0.1 \mathrm{wt} \%$ to 0.3 wt\%) of the analyzed Cr-spinels.

\subsection{Olivine and Pyroxenes}

Analyzed olivines are fairly homogenous in composition and do not show any distinct zoning from core to rim. No major

Fig. 7. (a) $\mathrm{Cr}$ spinel compositions from the Spontang ophiolite complex, in terms of $\mathrm{Cr} \#[\mathrm{Cr} /(\mathrm{Cr}+\mathrm{Al})]$ versus $\mathrm{Mg} \#\left[\mathrm{Mg} /\left(\mathrm{Mg}+\mathrm{Fe}{ }^{2+}\right)\right]$ overlain on the classification of the composition of $\mathrm{Cr}$-spinel and ferrian chromite in terms of $\mathrm{Cr} \#$ versus Mg\# diagram. Data for spinel in modern abys- sal peridotites is from Dick and Bullen (1984) and Juteau et al. (1990). Field for spinel in boninites is taken from Dick and Bullen (1984). Data for spinel in fore-arc peridotites are from Ishii et al. (1992) and Ohara and Ishii (1998). Cr-spinel composition is also contoured at a nominal temperature of $1200{ }^{\circ} \mathrm{C}$ for olivine compositions from Fo ${ }_{90}$ to Fog6 (quantitatively computed by Dick and Bullen, 1984). Arrows with ticks rep- resent the percentage of melting of the host peridotite after Hirose and Kawamoto, (1995). Black, pink and orange samples are from the Ker- guelen harzburgites (after Gregoire et al., 1997), South Sandwich peridotites (after Pearce et al., 2000) and Mariana Trough peridotites (after Ohara et al., 2002) respectively. (b) $\mathrm{Cr}_{2} \mathrm{O}_{3}$ versus $\mathrm{Al}_{2} \mathrm{O}_{3}$ in the spinels to determine the spinel origin. All samples plot in the mantle array field. The fields are from Conrad and Kay (1984), Haggerty (1989) and Kepezhinskas et al. (1995). (c) $\mathrm{Cr}_{1} \mathrm{Fe}^{3+}$ and Al relations in spinels of the Spon- tang peridotites. The superimposed fields are taken from Jan and Windley (1990). All analysed samples plot in the residual peridotites or ophiolites field in this diagram. (d) Compositional variations of orthopyroxene and clinopyroxene projected in the Di-En-Hd-Fs quadrilateral of the Spontang peridotites (after Morimoto et al., 1988). 

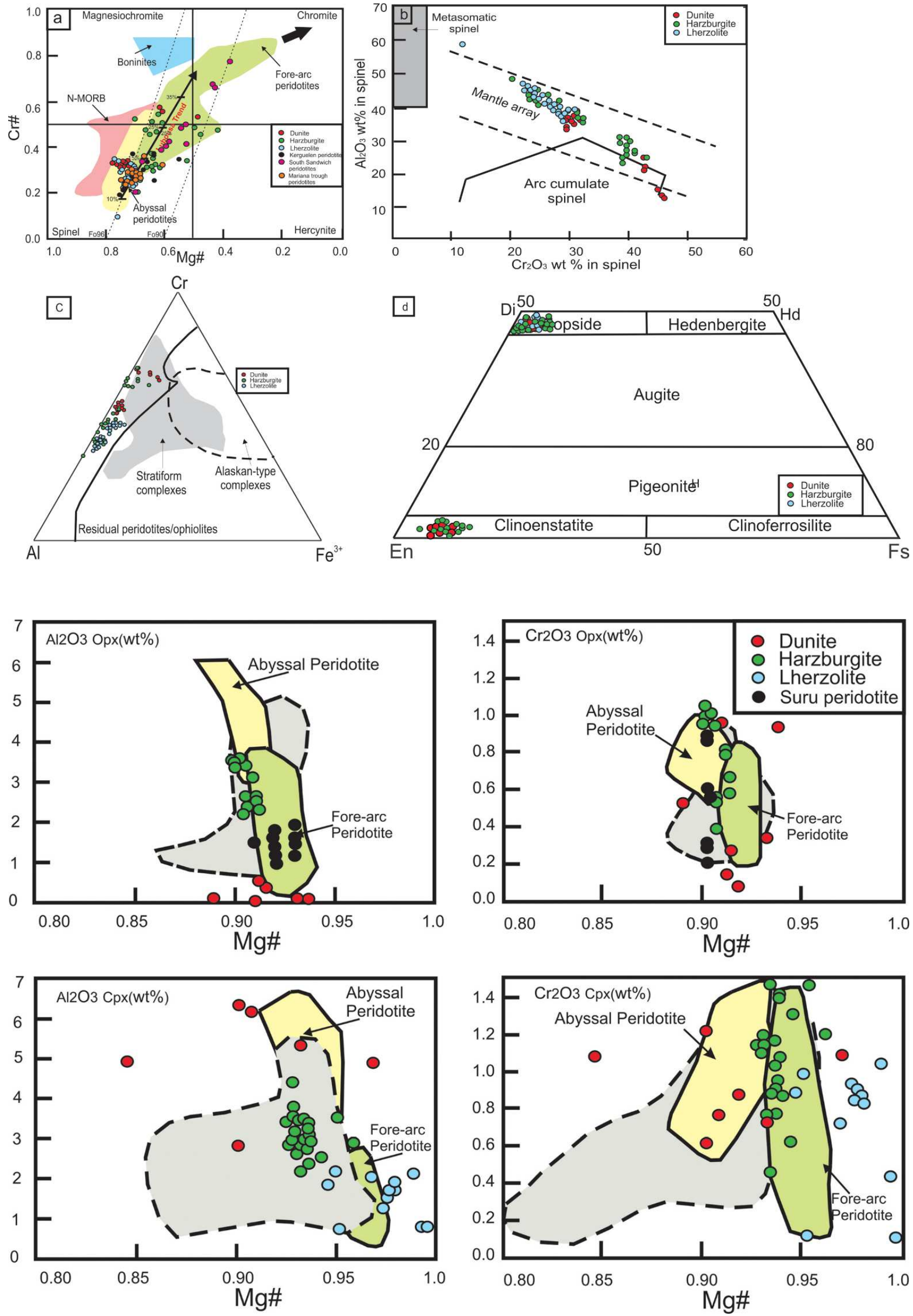
Fig. 8. Compositional variations of pyroxenes from peridotites of the Spontang ophiolite complex. $\mathrm{Cr}_{2} \mathrm{O}_{3}$ and $\mathrm{Al}_{2} \mathrm{O}_{3}$ vs. $\mathrm{Mg \#}$. Fields outline for clinopyroxene and orthopyroxene compositions in abyssal peridotites (Johnson et al., 1990) and forearc peridotites (Ishii et al., 1992). Dashed field outlines compositions in peridotites from the YZSZ ophiolites (Hébert et al., 2003; Dubois-Côté, 2004). Black dots are Suru per- idotites after Bhat et al. (2018).

variation in the composition is observed among the studied be a result of reactive melt percolation (Abily and peridotites. Forsterite content (Mg\#) in the analyzed crystals has a restricted compositional range between $87 \%$ and $92 \%$ in the lherzolite and harzburgite samples and between $89 \%$ and $91 \%$ in the dunite which is close to that of olivine in abyssal peridotites (average Fo content: 91\%; Dick and Bullen, 1984). $\mathrm{NiO}$ contents range between $0.21 \mathrm{wt} \%$ and $0.39 \mathrm{wt} \%$.

Analyzed pyroxenes in the investigated harzburgites consist

of enstatite orthopyroxene $\left(\mathrm{En}_{89-90} \mathrm{Wo}_{1-2} \mathrm{Fs}_{8-9}\right)$. Clinopyroxene in the lherzolites and harzburgites is diopsidic with compositions $\mathrm{En}_{50} \mathrm{Wo}_{49} \quad \mathrm{Fs}_{1}$ and $\mathrm{En}_{47} \mathrm{Wo}_{48} \mathrm{Fs}_{9}$ respectively (Fig. $7 \mathrm{~d}$ ) and do not exhibit any systematic zoning and are chemically homogenous. Clinopyroxenes from lherzolites show high Mg\# averaging 0.978. Harzburgite clinopyroxene shows Mg\# ranging between 0.926 and 0.959 averaging 0.935 when compared with orthopyroxene (Av: 0.905; 0.899-0.912). Cr\# values in enstatite are lower (0.1000.180 ) as compared with diopside (0.119-0.245). Dunitic samples contain orthopyroxene and clinopyroxene with the following compositions $\mathrm{En}_{8} \mathrm{Wo}_{88} \mathrm{Fs}_{6}$ and $\mathrm{En}_{7} \mathrm{Wo}_{70} \mathrm{Fs}_{26}$ respectively. Clinopyroxene in the dunites (Av: 0.942; 0.847-1.028) shows higher Mg\# as compared with orthopyroxene (Av: 0.9015; 0.890-0.937) and could

Ceuleneer,

2013; Rospabe et al., 2017). Overall clinopyroxenes have $\mathrm{Al}_{2} \mathrm{O}_{3}$ contents that range from $1.52 \mathrm{wt} \%$ to $5 \mathrm{wt} \%, \mathrm{Cr}_{2} \mathrm{O}_{3}$ in the range of $0.65 \mathrm{wt} \%$ to $1.27 \mathrm{wt} \%$. Orthopyroxnes have $\mathrm{Al}_{2} \mathrm{O}_{3}$ and $\mathrm{Cr}_{2} \mathrm{O}_{3}$ contents varying between 0.56 wt $\%$ to 3 wt $\%$ and 0.46 wt $\%$ to 0.56 wt $\%$ respectively. Orthopyroxene and clinopyroxene compositions for the harzburgites when plotted in the $\mathrm{Mg \#}$ vs. $\mathrm{Al}_{2} \mathrm{O}_{3}$ and $\mathrm{Cr}_{2} \mathrm{O}_{3}$ diagrams (Fig. 8), are observed to fall intermediate to the fields of abyssal and fore-arc peridotites. Dunite samples however, do not show any consistent trends. Lherzolite samples on the contrary show few samples falling in the fore-arc region. Chemical compositions of pyroxenes from the Spontang peridotites are compared with peridotites along the Indus Suture Zone viz. Suru peridotites (orthopyroxenes; Bhat et al., 2018) and the peridotites along the Yarlung Zangpo Suture Zone (Hébert et al., 2003; Dubois-Côté, 2004).

\subsection{Geothermobarometry}

Equilibration temperatures for the studied peridotite samples have been calculated using compositions from coexisting mantle phases and detailed calculations are given in Supplementary Tables S1 and S2. Care was taken to analyse orthopyroxene porphyroclasts free of clinopyroxene exsolutions lamellae. Using the partitioning of $\mathrm{Mg}$ and $\mathrm{Fe}^{2+}$ between olivine and spinel of Fabries (1979) the calculated temperatures range from $646^{\circ} \mathrm{C}$ to $795^{\circ} \mathrm{C}$. Application of the two-pyroxene geothermometer of Brey and Kohler (1990), yielded temperatures varying between $718{ }^{\circ} \mathrm{C}$ and $896^{\circ} \mathrm{C}$, assuming a pressure of $1.5 \mathrm{GPa}$ typical of upper mantle peridotites within the spinel stability field (Gasparik, 1987). The low temperature values yielded by olivine-spinel geothermometer may have resulted due to recrystallisation of different rates of diffusion between the mineral pairs used in the calculation. 


\section{DISCUSSION}

Spinel habits and their compositions can be used to understand the genesis of ultramafic rocks. Spinel's originating from the deeper upper mantle has been attributed to multiple origins ranging from products of partial melting to metasomatic processes and silicate decomposition (Haggerty, 1991). Ophiolitic spinels are widely considered to be derived from the upper mantle or as a product of crystal fractionation in cumulate sequences in the lithosphere (Paktunc, 1990). The spinels from the Spontang ophiolite along the Indus suture zone are compositionally consistent with the exception of some minor increase and decrease of certain elements. Morphological changes are observed in the spinel grains from symplectitic intergrowths in the harzburgites to more idiomorphic grains in the dunites. The $\mathrm{Cr} \#$ in the spinels from the harzburgites and dunites are observed to show an inverse relationship with $\mathrm{Al}$ and $\mathrm{Mg}$, i.e., with increasing $\mathrm{Cr}$ content the spinel gets depleted in $\mathrm{Al}$ and $\mathrm{Mg}$, whereas $\mathrm{Cr} \#$

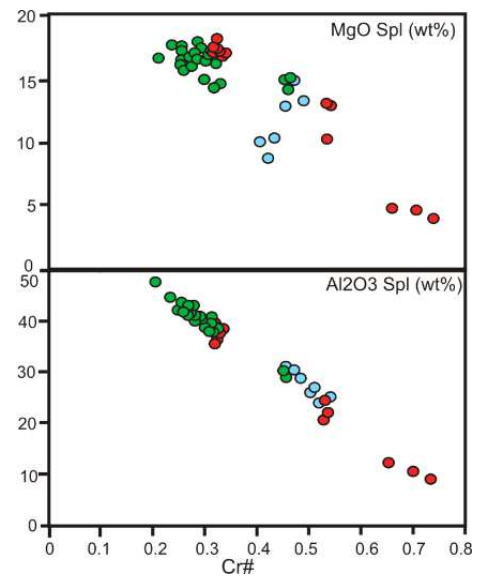

versus Mg in lherzolites show a positive correlation (Fig. 9) indicating that the lherzolites represent the fertile mantle. Spinel's evolved from an $\mathrm{Al}$ rich phase to $\mathrm{Cr}$ rich-Al poor phase which are expected as a result of partial melting (Karmalkar et al., 1997) coinciding with the above-mentioned morphological changes. Spinel-orthopyroxene intergrowth observed in the harzburgites could be result of garnet
+ olivine reaction indicating garnet to spinel peridotite transition during partial melting (Karmalkar et al., 1995, 1997).

\subsection{Interpretation of $\mathrm{Cr}$-spinel Data}

Compositions of accessory Cr-spinel in peridotites is regarded as a useful tool for revealing melting processes in mantle (e.g., Okamura et al., 2006; Uysal et al., 2007). Cr-spinel is known to be extremely sensitive to bulk composition mineralogy and petrogenesis of the host rock (Irvine, 1965, 1967; Evans and Frost, 1975; Dick, 1977b; Fisk and Bence, 1980). Cr-spinel compositions help determine the degree of partial melting of the mantle source and/or composition of produced mafic melt (e.g., Dick and Bullen, 1984; Arai, 1992; Zhou et al., 1998; Proenza et al., 1999; Barnes and Roeder, 2001; Hellebrand et al., 2001; Kamenetsky et al., 2001; Zhou et al., 2005; González-Jiménez et al., 2011) as well as processes involved in the evolution of upper mantle rocks i.e., mantle metasomatism (e.g., Kubo, 2002; Arif and Jan,

2006). It is known that $\mathrm{Al}$ and $\mathrm{Cr} \#$ of spinel is sensitive to mantle melting processes and that $\mathrm{Al}$ systematically decreases whereas Cr\# increases with the degree of peridotite depletion (e.g., Arai, 1994; Zhou et al., 2005; Zaccarini et al., 2011; Uysal et al., 2012). In the studied samples, the $\mathrm{Cr} \#$ of chromian spinel is observed to be lower in spinel from lherzolitic and harzburgitic rocks compared with dunites. In additions to this, $\mathrm{TiO}_{2}$ and $\mathrm{MnO}$ contents are low typical of unaltered Cr-spinel in ultramafic rocks (e.g., Barnes, 2000; Singh and Singh, 2013).

Differences among the studied peridotites can be observed in the plot of $\mathrm{Cr} \#$ in spinel versus Fo content in olivine (Fig. 10). The spinel $\mathrm{Cr} \#$ is observed to increase as the Fo content in olivine increases such that the spinel-olivine pairs from the studied peridotites lie within the olivinespinel mantle array (Arai, 1994). This correlation between the spinel and olivine compositions confirms the mantle residue origin of the investigated samples. In the $\mathrm{Cr} \#$ vs. Mg\# plot, Cr-spinels from the investigated peridotites fall mainly along a trend defined by a large increase in $\mathrm{Cr} \#$ for a small decrease in Mg\# (Hirose and Kawamoto, 1995) and follow the Luobusa trend defined by spinel of Yarlung Zangpo 
chemical variations observed in spinel caused by different degrees of apparent partial melting on the host peridotites. Spinels aligned along the Luobusa trend have a low $\mathrm{TiO}_{2}$ content $(<0.14 \mathrm{wt} \%)$ and are characterized by a small decrease in $\mathrm{TiO}_{2}$ for a larger increase in $\mathrm{Cr} \#$ and are consistent with observations made in the studied samples (Fig. 7a). Cr-spinel hosted in the lherzolites are represented by higher Mg\# and lower Cr\# suggesting up to

$10-12 \%$ partial melting (Fig. 10). On the other hand, harzburgites contain Cr-spinel with lower Mg\# and higher Cr\# up to $18-22 \%$ partial melting, whereas the dunites with highest $\mathrm{Cr} \#$ are observed to form a separate cluster indicating higher (25-28\%) degrees of partial melting consistent with those provided by Hirose and Kawamoto (1995) (Fig. 7a). Based on the above, the studied peridotites from the Spontang ophiolite are inferred to be produced by variable degrees of mantle melting and that the dunites represent a mantle residue resulting from higher degree of partial melting.

\subsection{Petrogenesis and Tectonic Setting}

Traditionally, ophiolites were believed to form along mid ocean ridge spreading centres however, recent studies of modern oceanic basins indicate that subduction is an important process involved in the formation of these ophiolites more commonly known as supra-subduction (SSZ) ophiolites. Globally, most well-preserved ophiolites appear to show mantle compositions of both MOR and SSZ (Pearce et al., 1984; Stern, 2004). Mantle

Fig. 10. $\mathrm{Cr} \#$ in spinel versus Fo content of olivine in peridotites of Spontang ophiolite. Fields for spinels occurring in abyssal (and ocean ridge), oceanic SSZ and passive margin peridotites are after Dick and Bullen (1984) and Pearce et al. (2000). OSMA means Olivine Spinel Mantle Array and fractionation line of boninites are after Arai (1994a, 1994b).

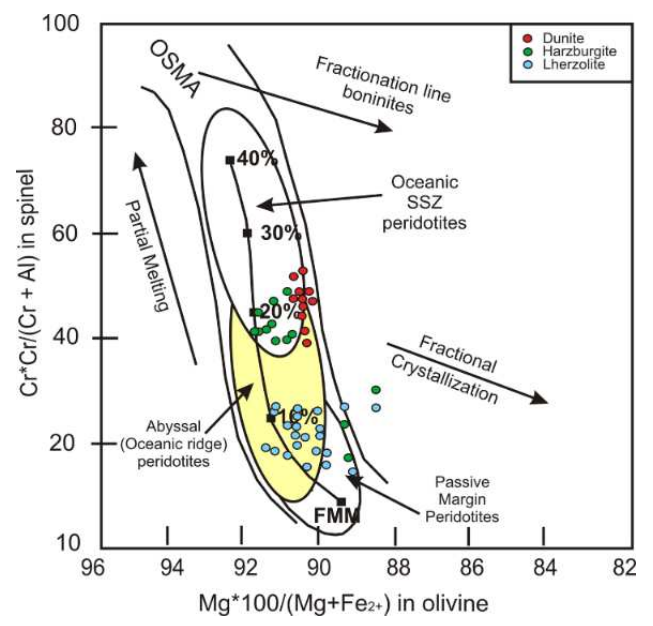

peridotites are known to contain both SSZ and abyssal peridotites in the fore-arc regions (Parkinson and Pearce, 1998; Pearce et al., 2000). Composition of Cr-spinel in addition to the elemental and modal composition of the mineral constituents of mantle peridotites is considered to be a good indicator of the tectono- magmatic history of the host rock. (e.g., Dick and Bullen, 1984; Arai, 1992; Zhou et al., 2005; Arai et al., 2011; Ahmed et al., 2012; Uysal et al., 2012), and can be used to determine the degree of partial melting (Dick and Bullen, 1984; Arai, 1994; Zhou et al., 1996; Hellebrand et al., 2002; Aswad et al., 2011 and references therein). SSZ peridotites are characterized by spinels with high Cr\#s ranging between 38 to 80 (e.g., Dick and Bullen, 1984; Juteau et al., 1990; Ishii et al., 1992; Ohara and Ishii, 1998) and pyroxenes with extremely low $\mathrm{Al}, \mathrm{Ti}$ and Na indicating significantly higher degrees of partial melting (> $15 \%$ ) as compared to abyssal peridotites with low Cr\# in spinel = 38 to 58 (Arai, 1994; Gaetani and Grove, 1998; Choi et al., 2008).

Petrography and geochemistry of the studied samples provide clear evidence of the Spontang peridotites having a multi-process history. The Spontang peridotites are characterized by increase in $\mathrm{Fo}$ and $\mathrm{NiO}$ contents of olivine (Fig. 11), $\mathrm{Mg \#}$ and $\mathrm{Cr}_{2} \mathrm{O}_{3}$ content of pyroxenes (Fig. 8), and $\mathrm{Cr} \#$ of spinel with decreasing $\mathrm{Al}_{2} \mathrm{O}_{3}$ (Fig. 9) and $\mathrm{TiO}_{2}$ (Fig. 12) content in spinel and bulk rock as melting progresses. Similar petrological and geochemical observations are reported in upper mantle peridotites of the YZSZ (Dupuis et al., 2005 and references therein) along with peridotites from other Himalayan ophiolites (Fig. 8; Bhat et al.,

2018); mantle xenoliths of the Kerguelen archipelago (Grégoire et al., 1997), and in peridotites of the South Sandwich arc-basin (Pearce et al., 2000) and Mariana Trough (Ohara et al., 2002) (Fig. 7a). The spinels compositions from studied harzburgites fall in the fore-arc region with lherzolites and dunites falling in the abyssal peridotite fields (Fig. 7a). The Spontang peridotites have olivine

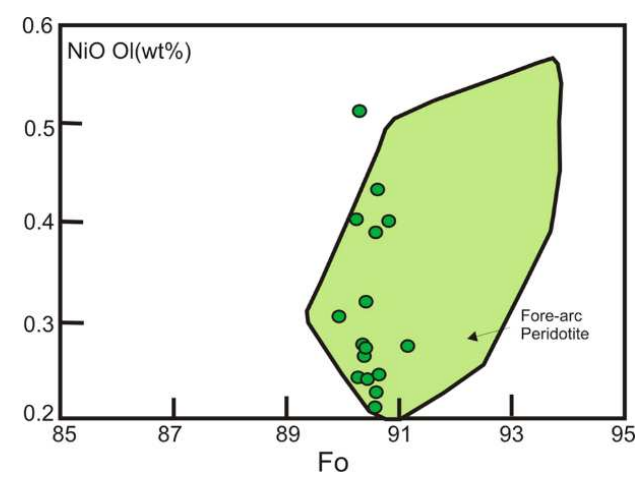

Fig. 11. Variation of $\mathrm{NiO}$ versus $\mathrm{Fo}$ in olivine from peridotites of the Spontang ophiolite complex. Field outline is olivine compositions in fore-arc peridotites (after Ishii et al., 1992). 


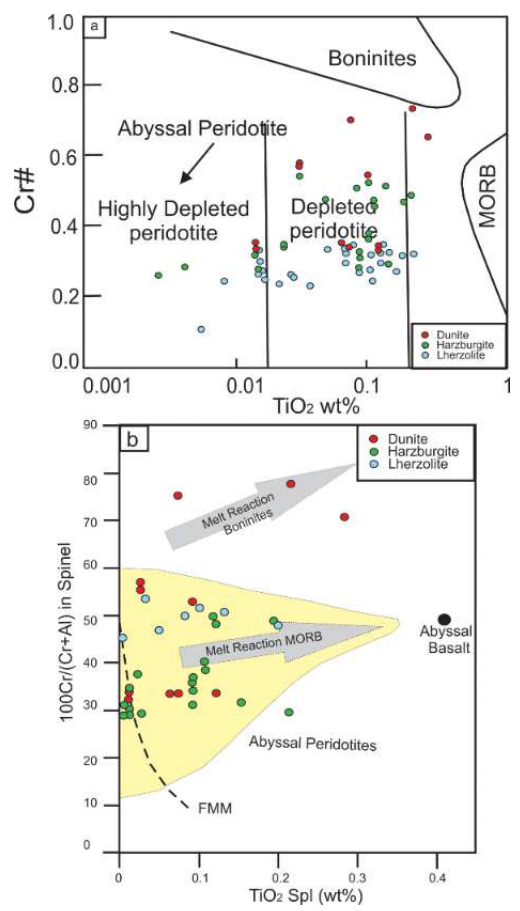

Fig. 12. (a) $\mathrm{TiO}_{2}$ vs. $\mathrm{Cr} \#$ diagram in spinels of Spontang ophiolite. Fields are after Dick and Bullen (1984); Jan and Windley (1990); Arai (1992). (b) Dotted line field of abyssal peridotite spinels (after Dick and Bullen, 1984); Dotted arrow shows effect of MORB melt on refractory abyssal peridotite spinels. Grey arrow shows effect of boninite melt reaction on refractory suprasubduction zone peridotite spinels. $\mathrm{FMM}=$ Fertile $\mathrm{MORB}$ Mantle.

clinopyroxene and orthopyroxene compositions similar to those from both abyssal and fore-arc peridotites (Fig. 6). The Crspinels when plotted in the $\mathrm{TiO}_{2}$ vs. Cr\# diagram (Fig. 12a) fall within the depleted peridotite field to highly depleted mantle fields typical of SSZ settings (Ohara et al., 2002) away from the MORB field. The $\mathrm{Al}_{2} \mathrm{O}_{3}$ vs. $\mathrm{TiO}_{2}$ diagram (Fig. 13) the samples plot between the MOR and SSZ peridotites. Overall, the mineral chemistry of the Spontang peridotites are characterized by interaction between depleted magma and ultra-depleted melts of pre-existing oceanic lithosphere, typical of suprasubduction zone settings (Fig. 12b; Choi et al., 2008).

Whole rock REE signatures also suggest formation of the Spontang peridotites in a supra-subduction zone setting (Fig.7). In comparison with the Spontang peridotites, Figure 7 shows peridotites found along the Indus-Yarlung Suture

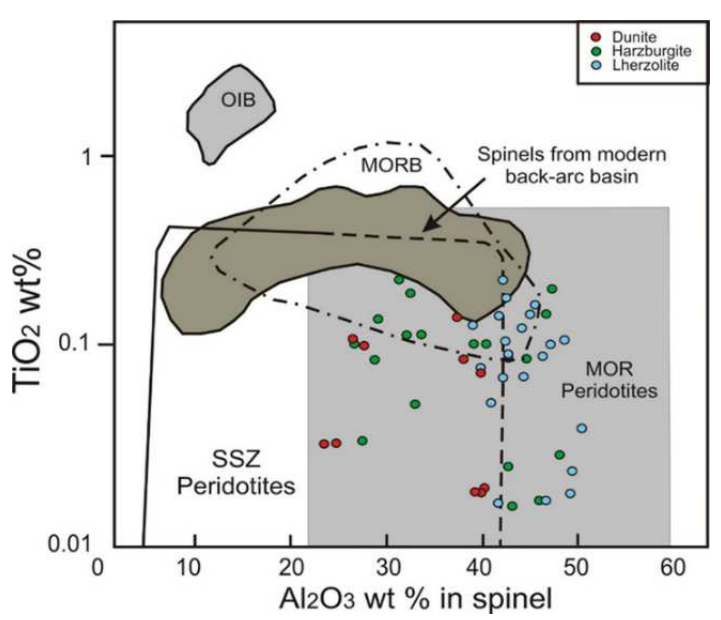

Fig. 13. $\mathrm{TiO}_{2}$ vs. $\mathrm{Al}_{2} \mathrm{O}_{3}$ of spinels from peridotites of the Spontang ophiolite complex. Fields are after Kamenetsky et al. (2001)

zone (Fig. 7; Dupuis et al., 2005 and references therein) and fore-arc peridotites from the Leg 125 peridotites from Western Pacific region (after Parkinson and Pearce, 1998) along with abyssal peridotites from the Manipur ophiolitic complex (after Singh, 2013). The studied samples have depleted LREE profiles similar to fore-arc peridotites in comparison to LREE's from abyssal settings that exhibit relatively flat to upward-inflected patterns (Fig. 6). LREE enrichment in sample E-5 could be attributed to interaction of melt or aqueous fluids (Rospabé et al., 2017,2018). Overall MREE-HREE profiles display positive slopes comparable with abyssal peridotites. Overall the studies samples display spoonshaped REE profiles characteristic of interaction between LREE-enriched melt, derived from the subducting slab and LREE-depleted mantle residues (Edwards, 1990; Kelemen et al., 1992; Zhou et al., 2005). Overall, based on the mineral and whole rock geochemistry, the Spontang ophiolite complex shows evidence of a mid-ocean ridge history prior to a suprasubduction history.

\section{CONCLUSIONS}

1) The ultramafic bodies of the Spontang ophiolite complex are presented by dunites, harzburgites and minor lherzolites displaying porphyroclastic to granular textures. Morphologically spinels in the studied samples occur as xenomorphic, vermicular intergrowths displaying characteristic holly leaf shapes. Euhedral spinel inclusions within olivine at places are also observed. Morphological changes in the spinel grains range from symplectitic intergrowths in the harzburgites to more idiomorphic grains in the dunites. Spinel's 
evolved from an $\mathrm{Al}$ rich phase to $\mathrm{Cr}$ rich-Al poor phase which are expected as a result of partial melting coinciding with the above-mentioned morphological changes.

2) The Spontang peridotites are characterized by increase in Fo and $\mathrm{NiO}$ contents of olivine, $\mathrm{Mg \#}$ and $\mathrm{Cr}_{2} \mathrm{O}_{3}$ content of pyroxenes, and $\mathrm{Cr} \#$ of spinel with decreasing $\mathrm{Al}_{2} \mathrm{O}_{3}$ and $\mathrm{TiO}_{2}$ content in spinel and bulk rock as melting progresses. $\mathrm{Cr} \#$ values of Cr-spinel increase and $\mathrm{Mg \#}$ values decrease from lherzolite to harzburgite and dunite. Studied spinels are characterized by a small decrease in $\mathrm{TiO}_{2}$ for a larger increase in $\mathrm{Cr} \#$ consistent with observations for spinels aligned along the Luobusa trend of the YZSZ ophiolites.

3) Mineral and whole-rock chemistry of the Spontang peridotites is characterized by interaction between magma and pre-existing oceanic lithosphere, typical of suprasubduction zone settings. The Spontang peridotites have olivine, clinopyroxene and orthopyroxene compositions similar to those from both abyssal and fore-arc peridotites with most spinels from harzburgites falling in the fore-arc region with lherzolites and dunites falling in the abyssal peridotite fields. Overall the studied samples display spoon shaped REE profiles characteristic of interaction between LREE-enriched melt, derived from the subducting slab and LREE-depleted mantle residues.

4) Equilibration temperatures for the studied peridotites calculated using the two-pyroxene geothermometer and the partitioning of $\mathrm{Mg}$ and $\mathrm{Fe}^{2+}$ between olivine and spinel yield temperatures varying between $707^{\circ} \mathrm{C}$ and $883{ }^{\circ} \mathrm{C}$ and $646^{\circ} \mathrm{C}$ to $795^{\circ} \mathrm{C}$ respectively typical of mantle peridotites within spinel stability field.

\section{ACKNOWLEDGMENTS}

The authors thank the Head, Department of Geology, SPPU for providing necessary facilities. MKJ acknowledges the financial support received from SERB by way of major research project under its Young Scientist Scheme (Ref No. SR/FTP/ES-2/2014) under which most of the spinel chemistry work was carried out and DST by way of its Women's scientist scheme (Ref No. SR/ WOS-A/EA14/2017).

\section{REFERENCES}

Abily, B. and Ceuleneer, G., 2013, The dunitic mantle-crust transition zone in the Oman ophiolite: residue of melt-rock interaction, cumu- lates from high-MgO melts, or both? Geology, 41, 67-70. https:// doi.org/10.1130/G33351.1

Ahmad, T., Tanaka, T., Sachan, H.K., Asahara, Y., Islam, R., and Khanna, P.P., 2008, Geochemical and isotopic constraints on the age and ori- gin of the Nidar Ophiolitic Complex, Ladakh, India: implications for the Neo-Tethyan subduction along the Indus suture zone. Tec- tonophysics, 451, 206-224.
Ahmed H.A., Harbi H.M., and Habtoor A.M., 2012, Compositional variations and tectonic settings of podiform chromitites and associ- ated ultramafic rocks of the Neoproterozoic ophiolite at Wadi Al Hwanet, northwestern Saudi Arabia. Journal of Asian Earth Sci- ences, 56, 118-134.

Aitchison, J.C., Badengzhu, Davis, A.M., Liu, J., Luo, H., Malpas, J., McDermid, I., Wu, H., Ziabrev, S., and Zhou, M.F., 2000, Remnants of a Cretaceous intra-oceanic subduction system within the Yarlung- Zangbo suture (southern Tibet). Earth and Planetary Science Let- ters, 183, 231-244.

Aitchison, J.C., McDermid, I.R.C., Ali, J.R., Davis, A.M., and Zyabrev, S.V., 2007, Shoshonites in southern Tibet record Late Jurassic rifting of a Tethyan intraoceanic island arc. The Journal of Geology, 115, 197-213.

Anonymous, 1972, Penrose field conference on ophiolites.

Geotimes, 17, 24-25.

Arai, S., 1992, Chemistry of chromian spinel in volcanic rocks as a potential guide to magma chemistry. Mineralogical Magazine, $56,173-184$

Arai, S., Okamura, H., Kadoshima, K., Tanaka, C., Suzuki, K., and Ishimaru, S., 2011, Chemical characteristics of chromian spinel in plutonic rocks: implications for deep magma processes and discrimination of tectonic setting. Island Arc, 20, 125-137.

Arai, S., 1994a, Characterization of spinel peridotites by olivinespinel compositional relationships: review and interpretation. Chemical Geology, 113, 191-204.

Arai, S., 1994b, Compositional variation of olivine-chromian spinel in Mg-rich magmas as a guide to their residual spinel peridotites. Jour- nal of Volcanological Geothermal Research, 59, 279-293.

Arif, M. and Jan M.Q., 2006, Petrotectonic significance of the chemistry of chromite in the ultramafic-mafic complexes of Pakistan. Journal of Asian Earth Sciences, 27, 628-646.

Aswad, K.J., Aziz, N.R., and Koyi, H.A., 2011, Cr-spinel compositions in serpentinites and their implications for the petrotectonic history of the Zagros Suture Zone, Kurdistan Region, Iraq. Geological Maga- zine, 148, 802-818.

Baker, M.B. and Beckett, J.R., 1999, The origin of abyssal peridotites:

a reinterpretation of constraints based on primary bulk compositions.

Earth and Planetary Science Letters, 171, 49-61.

Barrat, J.A., Keller, F., Amosse, J., Taylor, R.N., Nesbitt, R.W., and Hirata, T., 1996, Determination of rare earth elements in sixteen silicate refer- ences by ICP-MS after Tm addition and ion exchange separation. Geostandard Newsletter, 20, 133-139.

Barrat, J.A., Zanda, B., Moynier, F., Bollinger, C., Liorzou, C., and Bayon, G., 2012, Geochemistry of CI chondrites: major and trace elements, and $\mathrm{Cu}$ and $\mathrm{Zn}$ isotopes. Geochimica et Cosmochimica Acta, 83, 79-92.

Barnes, S.J. and Roeder, P.L., 2001, The range of spinel compositions in terrestrial mafic and ultramafic rocks. Journal of Petrology, $42,2279-2302$.

Barnes S.J., 2000, Chromite in komatiites. II. Modification during greenschist to mid-amphibolite facies metamorphism. Journal of Petrology, 41, 387-409.

Bhat, I.M., Ahmad, T., and Subba Rao, D.V., 2018, Origin and evolution of Suru Valley ophiolite peridotite slice along Indus suture zone, 
Ladakh Himalaya, India: implications on melt-rock interaction in a subduction-zone environment, Chemie der Erde. https://doi.org/10.1016/j.chemer.2018.103

Bodinier, J.L. and Godard, M., 2007, Orogenic, ophiolitic and abyssal peridotites. In: Holland, H.D. and Turekian, K.K. (eds.), Treatise on Geochemistry. Elsevier, Amsterdam, 2, p. 1-73. https://doi.org/10.1016/B0-08-043751-6/02004-1

Bonatti, E. and Michael, P.J., 1989, Mantle peridotites from continental rifts to oceanic basins to subduction zones. Earth Planetary Sci- ence Letters, 91, 297-311.

Brey, G.P. and Köhler, T., 1990, Geothermobarometry in four-phase lherzolites. II. New thermobarometers and practical assessment of existing thermobarometers. Journal of Petrology, 31, 13531378.

Choi, S.H., Shervais, J.W., and Mukasa, S.B., 2008, Supra-subduction and abyssal mantle peridotites of the Coast Range ophiolite, Cali- fornia. Contributions to Mineralogy and Petrology, 156, 551-576. https://doi.org/10.1007/s00410-008-0300-6

Colchen, M. and Reuber, I., 1986, Les mélanges ophiolitiques du

Zanskar. Himalaya du Ladakh. Comptes rendus de l'Académie des

Sciences, Paris, 303, 719-724.

Colchen, M., Reuber, I., Bassoullet, J.R., Bellier, J.R., Blondeau, A., Lys, M., and de Wever, P., 1987, Données biostratigraphiques sur les mélanges ophiolitiques du Zanskar, Himalaya du Ladakh. Comptes rendus de l'Académie des Sciences, Paris, 305, 403-406. Conrad, W.K. and Kay, R.W., 1984, Ultramafic and mafic inclusions from Adak Island: crystallisation history and implications for the nature of primary magmas and crustal evolution in the Aleutian arc. Journal of Petrology, 25, 88-125. Corfield, R.I., Searle, M.P., and Green, O.R., 1999, Photang thrust sheet- An accretionary complex structurally below the Spontang ophio- lite constraining timing and tectonic environment of ophiolite obduction, Ladakh Himalaya, NW India. Journal of the Geological Society, 156, 1031-1044.

Corfield, R.I., Searle, M.P., and Pedersen, R.B., 2001, Tectonic setting, origin, and obduction history of the Spontang ophiolite, Ladakh Himalaya, NW India. Journal of Geology, 109, 715-736. Deitrich, V.J., Frank, W., and Honegger, K., 1983, A JurassicCretaceous island arc in the Ladakh-Himalayas. Journal of Volcanology and Geothermal Research, 18, 405-433.

Dick, H.J.B., 1977a, Evidence of partial melting in the Josephine peri-dotite. Magma Genesis, 96, 59-62.

Dick, H.J.B., 1977b, Partial melting in the Josephine Peridotite I, the effect on mineral composition and its consequence for geobarom- etry and geothermometry. American Journal of Science, 277, 801- 832.

Dick, H.J.B. and Bullen, T., 1984, Chromium spinel as a petrogenetic indicator in abyssal and alpine-type peridotites and spatially associ- ated lavas. Contributions to Mineralogy and Petrology, 86, 54-76.

Dubois-Côté, V., 2004, Pétrologie et géochimie des ophiolites de la

Zone de Suture du Yarlung Zangbo (ZSYZ), Tibet: implications géody- namiques. M.Sc Thesis, Université Laval, Quebec City, 231 p. Dupuis, C., Hebert, R., Dubois-Côté, V., Guilmette, C., Wang, C.S., Li, Y.L., and Li, Z.J., 2005, The Yarlung Zangbo Suture Zone ophiolitic melange (Southern Tibet): new insights from geochemistry of ultra- mafic rocks. Journal of Asian Earth Sciences, 25, 937-960.
Edwards, S.J., 1990, Harzburgites and refractory melts in the Lewis hills massif, Bay of Island ophiolite complex: the base-metals and pre- cious-metals story. Canadian Mineralogist, 28, 537-552.

Evans, B.W. and Frost, B.R., 1975, Chrome-spinel in progressive meta- morphism. A preliminary analysis. Geochimica et Cosmochimica Acta, 39, 959-972.

Fabries, I., 1979, Spinel-olivine geothermometry in peridotites from ultramafic complexes. Contributions to Mineralogy and Petrology, 69, 329-336.

Fisk, M.R. and Bence, A.E., 1980, Experimental crystallization of chrome spinel in FAMOUS basalt 527-1-1. Earth Planetary Science Letters, 48, 111-123.

Frank, W., Gansser, A., and Trommsdroff, V., 1977, Geological observations in the Ladakh area (Himalayas). A preliminary report. Sch- weizerische Mineralogische und Petrographische Mitteilungen, 57, 89-113.

Fuchs, G., 1982, The Geology of Western Zanskar. Jahrbuch Geologisches Bundesanstalt, Vienna, 125, 50 p.

Gansser, A., 1974, The ophiolitic mélange, a world-wide problem on Tethyan examples. Eclogae Geologicae Helvetiae, 67, 479-507.

Gasparik, T., 1987, Orthopyroxene thermometry in simple and complex systems. Contributions to Mineralogy and Petrology, 96, 357-370.

Gaetani, G.A. and Grove, T.L., 1998, The influence of water on melting of mantle peridoite. Contributions to Mineralogy and Petrology, 131, 323-346.

Girardeau, J., Marcoux, J., and Zao, Y., 1984, Lithologic and tectonic environment of the Xigaze ophiolite (Yarlung Zangbo suture zone, Southern Tibet, China): kinematics of its emplacement. Eclogea Geologicae Helvetiae, 77, 153-170.

Girardeau, J., Mercier, J-C.C., and Wang, X., 1985a, Petrology of the mafic rocks of the Xigaze ophiolites, Tibet: implications for the genesis of the oceanic lithosphere. Contributions to Mineralogy and Petrol- ogy, 90, 309-321.

Girardeau, J., Mercier, J.-C.C., and Zao, Y., 1985b, Structure of the Xigaze ophiolite, Yarlung Zangbo suture zone, southern Tibet, China: genetic implications, Tectonics, 4, 267-288.

Godard, M., Jousselin, D., and Bodinier, J. L., 2000, Relationships between geochemistry and structure beneath a palaeospreading centre: a study of the mantle section in the Oman ophiolite. Earth Planetary Science Letters, 180, 133-148.

González-Jiménez, J.M., Proenza, J.A., Gervilla, F., Melgarejo, J.C., Blanco-Moreno, J.A., Ruiz-Sánchez, R., and Griffin, W.L., 2011, High-Cr and high-Al chromitites from the Sagua de Tánamo district, Mayarí-Cristal ophiolitic massif (eastern Cuba): constraints on their origin from mineralogy and geochemistry of chromian spinel and platinum-group elements. Lithos, 125, 101-121.

Göpel, C., Allègre, C.J., and Xu, R.H., 1984, Lead isotopic study of the Xigaze ophiolite (Tibet): the problem of the relationship between magmatites (gabbros, dolerites, lavas) and tectonites (harzburgites). Earth and Planetary Science Letters, 69, 301-310.

Grégoire, M., Lorand, J.P., Cottin, J.Y., Giret, A., Mattielli, N., and Weis, D., 1997, Xenoliths evidence for a refractory oceanic mantle perco- lated by basaltic melts beneath the Kerguelen archipelago. Euro- pean Journal of Mineralogy, 9, 1085-1100. 
Haggerty, S.E., 1989, Upper mantle opaque mineral stratigraphy and the genesis of metasomatites and alkali-rich melts. In: Ross, J. (ed.), Kimberlites and Related Rocks: 2. Their Mantle/Crust Setting, Dia- monds and Diamond Exploration. Geological Society of Australia, Special Publications, 14, p. 687-699.

Haggerty, S.E., 1991, Oxide mineralogy of the upper mantle. Reviews in Mineralogy, 25, 355-416.

Hébert, R., Huot, F., Wang, C.S., and Liu, Z.F., 2003, Yarlung Zangbo ophiolites, southern Tibet revisited: geodynamic implications from the mineral record. In: Dilek, Y. and Robinson, P.T., (eds.), Ophio- lites in Earth History. Geological Society, London, Special Publications,

218, p. 165-190. https://doi.org/10.1144/GSL.SP.2003.218.01.10

Hellebrand. E., Snow, J.E., Dick, H.J.B., and Hofmann, A.W., 2001, Coupled major and trace elements as indicator of the extent of melting in mid-ocean-ridge peridotites. Nature, 410, 677-681.

Hellebrand, E., Snow, J.E., Hoppe, P., and Hofmann, A., 2002, Garnetfield melting and late stage refertilization in 'residual' abyssal peri- dotites from the Central Indian Ridge. Journal of Petrology, 43, 2305-2338.

Hirose, K. and Kawamoto, T., 1995, Hydrous partial melting of lherzo- lite at $1 \mathrm{Gpa}$ : the effect of $\mathrm{H}_{2} \mathrm{O}$ on the genesis of basaltic magmas. Earth and Planetary Science Letters, 133, 463-473.

Honegger, K., Dietrich, V., Frank, W., Gansser, A., Thoni, M., and Trommsdorff, V., 1982, Magmatism and metamorphism in the Ladakh Himalayas (the Indus-Tsangpo Suture Zone). Earth and Planetary Science Letters, 60, 253-292.

Irvine, T.N., 1965, Chromian spinel as a petrogenetic indicator; Part 1, Theory. Canadian Journal of Earth Science, 2, 648-671.

Irvine, T.N., 1967, Chromian spinel as a petrogenetic indicator; Part 2, Petrologic Applications. Canadian Journal of Earth Science, 4, 71-103.

Ishii, T., Robinson, P.T., Maekawa, H., and Fiske, R., 1992, Petrological studies of peridotites from diapiric serpentinite seamounts in the Izu-Mariana fore-arc, Leg 125. In: Fryer, P., Pearce, J.A., and Stok- king, L.B. (eds.), Proceedings of the Ocean Drilling Program. Scientific Results, College Station, 125, p. 445-485. https://doi.org/10.2973/ odp.proc.sr.125.129.1992

Jan, M.Q., Windley, B.F., and Khan, A., 1985, The Waziristan ophiolite, Pakistan - General geology and chemistry of chromite and associ- ated phases. Economic Geology, 80, 294-306.

Jan, M.Q. and Windley, B.F., 1990, Chromian spinel-silicate chemistry in ultramafic rocks of the Jijal complex, northwest Pakistan, Jour- nal of Petrology, 31, 667-715.

Johnson, K.T.M., Dick, H.J.B., and Shimizu, N., 1990, Melting in the oceanic upper mantle: an ion microprobe study of diopsides in abyssal peridotites. Journal of Geophysical Research, 95, 26612678.

Juteau, T., Berger, E., and Cannat, M., 1990, Serpentinized, residual mantle peridotites from the M.A.R. median valley, ODP hole 670A $\left(21^{\circ} 10^{\prime} \mathrm{N}, 45^{\circ} 02^{\prime} \mathrm{W}\right)$ : primary mineralogy and geothermometry. In: Detrick, R., Honnorez, J., Bryan, W.B., and Juteau, T. (eds.), Pro- ceedings of the Ocean Drilling Program. Scientific Results, College Station, 106, p. 27-45. https://doi.org/10.2973/odp.proc.sr.106109.117.1990

Kakar, M.I., Mahmood, K., Khan, M., Kasi, A.K., and Manan, R.A., 2013, Petrology and geochemistry of gabbros from the Muslim Bagh Ophio- lite: implications for their petrogenesis and tectonic setting. Journal of
Himalayan Earth Sciences, 46, 19-

30.

Kamenetsky, V.S., Crawford, A.J., and Meffre, S., 2001, Factors controlling chemistry of magmatic spinel: an empirical study of associ- ated olivine, Cr-spinel and melt inclusions from primitive rocks. Journal of Petrology, 42, 655-671.

Karmalkar, N.R., Dessai, A.G., Duraiswami, R.A., and Leblanc, M., 1995, Evidence of garnet to spinel peridotite transition in the harz- burgites of the Indus ophiolite belt: an indication of their mantle origin. Current Science, 69, 767-770.

Karmalkar, N.R., Dessai, A.G., and Duraiswami, R.A., 1997, Morphological and chemical changes in spinels and their bearing on the cumulate or residual nature of the peridotites from the Indus ophi- olite, India. The Indian Mineralogist, 31, 12-23.

Kelemen, P.B. and Sonnenfeld, M.D., 1983, Stratigraphy, structure, petrology and local tectonics, Central Ladakh, NW Himalaya. Sch- weizerische Mineralogische und Petrographische Mitteilungen, 63, 267-287.

Kelemen, P.B., Reuber, I., and Fuchs, G., 1988, Structural evolution and sequence of thrusting in the High Himalayan, TibetanTethys and Indus Suture zones of Zanskar and Ladakh, Western Himalaya. Journal of Structural Geology, 10, 129-130.

Kelemen, P.B., 1990, Reaction between ultramafic rock and

fractionating basaltic magma I. phase relations, the origin of calc-

alkaline magma series, and the formation of discordant dunite.

Journal of Petrol- ogy, 31, 51-98.

Kelemen, P.B., Dick, H.J.B., and Quick, J.E., 1992, Formation of harzburgite by pervasive melt/rock reaction in the upper mantle. Nature, 358, 635-641.

Kelemen, P.B., Hirth, G., Shimizu, N., Spiegelman, M., and Dick, H.J.B., 1997, A review of melt migration processes in the adiabatically upwell- ing mantle beneath oceanic spreading ridges. Philosophical Trans- actions of the Royal Society of London, 355, 283-318.

Kepezhinskas, P.K., Defant, M.J., and Drummond, M.S., 1995, Na metasomatism in the island-arc mantle by slab melt-peridotite interac- tion: evidence from mantle xenoliths in the north Kamchatka arc. Journal of Petrology, 36, 1505-1527. https://doi.org/10.1093/oxfordjour- nals.petrology.a037263

Kubo, K., 2002, Dunite formation processes in highly depleted peridot- ite: case study of the Iwanaidake peridotite, Hokkaido, Japan. Jour- nal of Petrology, 43, 423-448.

Lippard, S.J., Shelton, A.W., and Gass, I.G. (eds.), 1986, The Ophiolites of Northern Oman. Geological Society, London, Memoirs, 11, Boston, $178 \mathrm{p}$.

Maheo, G., Bertrand, H., Guillot, S., Villa, I.M., Keller, F., and Capiez, P., 2004, The South Ladakh ophiolites (NW Himalaya, India): an intra- oceanic tholeiitic arc origin with implication for the closure of the Neo-Tethys. Chemical Geology, 203, 273-303.

McDermid, I.R.C., Aitchison, J.C., Davis, A.M., Harrison, T.M., and Grove, M., 2002, The Zedong Terrane: a Late Jurassic intra-oceanic magmatic arc within the Yarlung-Tsangpo Suture Zone, Southeastern Tibet. Chemical Geology, 187, 267-277.

Mercier, J.C.C. and Nicolas, A., 1975, Textures and fabrics of uppermantle peridotites as illustrated by xenoliths from basalts. Journal of Petrology, 16, 454-487.

Miller, C., Thoeni, M., Frank, W., Schuster, R., Melcher, F., Meisel, T., 
and Zanetti, A., 2003, Geochemistry and tectonomagmatic affinity of the Yungbwa Ophiolite, SW Tibet. Lithos, 66, 155-172.

Morimoto, N., Fabries, J., Ferguson, A.K., Ginzburg, I.V., Ross, M., Seifert, F.A., Zussman, J., Aoki, K., and Gottardi, G., 1988, Nomenclature of pyroxenes. American Mineralogist, 73, 1123-1133.

Nicolas, A., Girardeau, J., Marcoux, J., Dupré, B., Wang, X.B., Cao, Y.G., Zeng, H.X., and Xiao, X.C., 1981, The Xigaze ophiolite (Tibet): a peculiar oceanic lithosphere. Nature, 294, 414-417.

Niu, Y., Langmuir, C.H., and Kinzler, R.J., 1997, The origin of abyssal peridotites: a new perspective. Earth and Planetary Science Letters, 152, 251-265.

Ohara, Y. and Ishii, T., 1998, Peridotites from the southern Mariana forearc: heterogeneous fluid supply in the mantle wedge. Island Arc, 7, 541-558.

Ohara, Y., Stern, R.J., Ishii, T., Yurimoto, H., and Yamazaki, T., 2002, Peridotites from the Mariana trough: first look at the mantle beneath an active back-arc basin. Contributions to Mineralogy and Petrol- ogy, 143, 1-18.

Ozawa, K., 1989, Stress-induced Al-Cr zoning of spinel in

deformed peridotites. Nature, 338, 141-

144https://doi.org/10.1038/338141a0

Paktunc, A.D., 1990, Origin of podiform chromite deposits by multistage melting, melt segregation and magma mixing in the upper mantle. Ore Geology Review, 5, 211-222.

Parkinson, I.J. and Pearce, J.A., 1998, Peridotites from the Izu-BoninMariana forearc (ODP Leg 125): evidence for mantle melting and melt-mantle interactions in a supra-subduction zone setting. Jour- nal of Petrology, 39, 1577-1618. https://doi.org/10.1093/petrology/39.9.1577

Pearce, J.A., Lippard, S.J., and Roberts, S., 1984, Characteristics and tec- tonic significance of supra-subduction ophiolites. In: Kokelaar, B.P. and Howells, M.F. (eds.), Marginal Basin Geology. Geological Soci- ety, London, Special Publications, 16, p. 777-794.

Pearce, J.A., Barker, P.F., Edwards, S.J., Parkinson, I.J., and Leat, P.T., 2000, Geochemistry and tectonic significance of peridotites from the South Sandwich arc-basin system, South Atlantic. Contribu- tions to Mineralogy and Petrology, 139, 36-53.

Pedersen, R.B., Searle, M.P., and Corfield, R.I., 2001, U-Pb Zircon ages from the Spontang ophiolite, Ladakh Himalaya. Journal of the Geological Society, 158, 513-520.

Proenza, J.A., Gervilla, F., Melgarejo, J.C., and Bodinier, J.L., 1999, Al and $\mathrm{Cr}$-rich chromitites from the Mayarí-Baracoa ophiolitic belt (eastern Cuba): consequence of interaction between volatile-rich melts and peridotites in suprasubduction mantle. Economic Geol- ogy, 94, 547-566.

Reuber, I., Colchen, M., and Mevel, C., 1992, The Spontang ophiolite and ophiolitic mélanges of the Zanskar, N.W. Himalaya, tracing the evolution of the closing Tethys in the upper Cretaceous to the Early Tertiary. In: Sinha, A.K. (ed.), Himalayan Orogen and Global Tec- tonics. International Lithosphere Program, A.A. Balkema, Rotter- dam, 197, p. 235-266.

Reuber, I., 1986, Geometry of accretion and oceanic thrusting of the Spontang ophiolite. Ladakh Himalaya. Nature, 321, 592-596.

Reibel, G. and Reuber, I., 1982, La klippe ophiolitique de

Spongtang- Photaksàr (Himalaya du Ladakh); une ophiolite sans cumulais. Comptes rendus de l'Académie des Sciences, 294, 557562.
Robertson, A., 2000, Formation of mélanges in the Indus Suture Zone, Ladakh Himalaya by successive subduction-related, collisional, postcollisional processes during Late Mesozoic-Late Tertiary time. In: Khan, M.A., Treolar, P.J., Searle, M.P., and Jan, Q. (eds.), Tecton- ics of the Nangat Parbat Syntaxis and the Western Himalaya. Geo- logical Society, London, Special Publications, 170 , p. 333-374. https://doi.org/10.1144/GSL.SP.2000.170.01.19

Rospabe, M., Ceuleneer, G., Benoit, M., Abily, B., and Pinet, P., 2017, Origin of the dunitic mantle-crust transition zone in the Oman ophiolite: the interplay between percolating magmas and high tem- perature hydrous fluids. Geology, 45, 471-474.

Rospabe, M., Benoit, M., and Candaudap, F., 2018, Determination of trace element mass fractions in ultramafic rocks by HR-ICP- MS: a combined approach using a direct digestion/dilution method and pre-concentration by co-precipitation. Geostandard Geoanalytical Research, 42, 115-129.

Rospabe, M., Benoit, M., Ceuleneer, G., Hodel, F., and Kaczmarek, MA., 2018, Extreme geochemical variability through the dunitic tran- sition zone of the Oman ophiolite: implications for melt/fluid-rock reactions at Moho level beneath oceanic spreading centers. Geo- chimica et Cosmochimica Acta, 234, 123.

Sarwar, G., 1992, Tectonic setting of the Bela Ophiolites, southern

Pakistan. Tectonophysics, 207, 359-381.

Searle, M.P., 1986, Structural evolution and sequence of thrusting in the High Himalayan, Tibetan-Tethys and Indus suture zones of Zanskar and Ladakh, Western Himalaya. Journal of Structural Geology, 8, 923-936.

Searle, M.P., Cooper, D.J.W. and Rex, A.J., 1988, Collision tectonics of the Ladakh-Zanskar Himalaya. Philosophical Transactions of the Royal Society of London, 326,117-150

Singh, A.K., 2013, Petrology and geochemistry of abyssal peridotites from the Manipur ophiolite complex, Indo-Myanmar Orogenic Belt, Northeast India: implication for melt generation in mid-oce- anic ridge environment. Journal of Asian Earth Science, 66, 258-276. https://doi.org/10.1016/j.jseaes.2013.02.004

Singh, A.K. and Singh, R.B., 2013, Genetic implications of Zn- and Mnrich Cr-spinels in serpentinites of the Tidding Suture Zone, eastern Himalaya, NE India. Geological Journal, 48, 22-38.

Stern, R.J., 2004, Subduction initiation: spontaneous and

induced. Earth and Planetary Science Letters, 226, 275-292.

Srikantia, S.V. and Razdan, M.L., 1981, Shilakong ophiolite nappe of Zanskar Mountains, Ladakh Himalaya. Journal of the Geological Soci- ety of India, 22, 227-234.

Uysal, I., Kaliwoda, M., Karsli, O., Tarkian, M., Sadiklar, M.B., and Ottley, C.J., 2007, Compositional variations as a result of partial melting and melt-peridotite interaction in an upper mantle section from the Ortaca area, southwestern Turkey. Canadian Mineralogist, 45, 1471-1493.

Uysal, I., Ersoy, E.Y., Karsl, O., Dilek, Y., Sadıklar, M.B., Ottley, C.J., Tiepolo, M., and Meisel, T., 2012, Coexistence of abyssal and ultradepleted SSZ type mantle peridotites in a Neo-Tethyan Ophiolite in SW Turkey: constraints from mineral composition, whole-rock geochemis- try (major-trace-REE-PGE), and Re-Os isotope systematics. Lithos, 132, 50-69.

Xia, B., Yu, H.X., Chen, G.W., Qi, L., Zhao, T.P., and Zhou, M.F., 2003, 
Geochemistry and tectonic environment of the Dagzhuka ophio- lite in the Yarlung-Zangbo suture zone, Tibet. Geochemical Jour- nal, 37, 311-324.

Yokoyama, T., Makishima, A., and Nakamura, E., 1999, Evaluation of the co-precipitation of incompatible trace elements with fluoride during silicate rock dissolution by acid digestion. Chemical Geol- ogy, 157, 175-187.

Zaccarini, F., Garuti, G., Proenza, J.A., Campos, L., Thalhammer, O.A., Aiglsperger, T., and Lewis, J.F., 2011, Chromite and platinum group elements mineralization in the Santa Elena Ultramafic Nappe (Costa Rica): geodynamic implications. Geologica Acta, 9, 407-423. https://doi.org/10.1344/105.000001696

Zaigham, N.A. and Mallick, K.A., 2000, Bela ophiolite zone of southern Pakistan: tectonic setting and associated mineral deposits. Geo- logical Society of America Bulletin, 112, 478-489.

Zhou, M.F., Robinson, P.T., and Malpas, J., 1996, Podiform chromitites in the Luobusa ophiolite (southern Tibet): implications for melt- rock interaction and chromite segregation in the upper mantle. Journal of Petrology, 37, 3-21.

Zhou, M. and Robinson, P.T., 1997, Origin and tectonic environment of podiform chromite deposits. Economic Geology, 92, 259-262. Zhou, M.F., Sun, M., Keays, R.R., and Kerrich, R.W., 1998, Controls on platinum-group elemental distributions of podiform chromitites: case study of high-Cr and high-Al chromitites from Chinese oro- genic belts. Geochimica et Cosmochimica Acta, 62, 677688.

Zhou, M.F., Robinson, P.T., Malpas, J., Edwards, S.J., and Qi, L., 2005, REE and PGE geochemical constraints on the formation of dun- ites in the Luobusa Ophiolite, southern Tibet. Journal of Petrology,

46, 615-639.

Publisher's Note Springer Nature remains neutral with regard to jurisdic- tional claims in published maps and institutional affiliations. 